\title{
The impact of stellar duplicity on planet occurrence and properties
}

\section{Observational results of a VLT/NACO search for stellar companions to 130 nearby stars with and without planets ${ }^{\star, \star \star}$}

\author{
A. Eggenberger ${ }^{1,2}$, S. Udry ${ }^{1}$, G. Chauvin ${ }^{3,2}$, J.-L. Beuzit ${ }^{2}$, A.-M. Lagrange ${ }^{2}$, D. Ségransan ${ }^{1}$, and M. Mayor ${ }^{1}$ \\ 1 Observatoire de Genève, Université de Genève, 51 ch. des Maillettes, 1290 Sauverny, Switzerland \\ e-mail: Anne.Eggenberger@obs.ujf-grenoble.fr \\ 2 Laboratoire d'Astrophysique de Grenoble, Université Joseph Fourier, BP 53, 38041 Grenoble Cedex 9, France \\ 3 European Southern Observatory, Alonso de Cordova 3107, Casilla 19001, Santiago 19, Chile
}

Received 9 March 2007 / Accepted 4 June 2007

\begin{abstract}
Context. Although it is commonly agreed that the presence of a close stellar companion is likely to affect planet formation and evolution, the precise effects and their actual impact on planet occurrence are still debated. Different conclusions have been reached on the theoretical side, while observational constraints are sparse, a consequence of the discrimination against close binaries in Doppler planet searches. Accordingly, basic questions such as how hospitable binaries are to planets and how binary separation and mass ratio impact on planet formation, remain poorly known.

Aims. In an effort to bring observational constraints on the occurrence and properties of planets in binaries and multiple stars, we have been conducting a dedicated investigation, the results of which will be presented in this series.

Methods. Our investigation follows two different approaches, one based on radial-velocity monitoring, the other based on direct imaging. In this first paper, we present the observational results from our systematic adaptive optics search with VLT/NACO for close stellar companions to 130 nearby stars, 57 with planets and 73 without, for comparison. The inclusion of a control sub-sample is a unique feature of our program that will enable a meaningful and rigorous comparison between the properties of planet-host stars and the properties of field stars subject to the same selection effects against close binaries, but showing no evidence for planetary companions.

Results. Our data reveal 95 companion candidates found in the vicinity of 33 of our targets. Nineteen of these candidates are true companions and 2 are likely bound objects. Among planet-host stars, we discovered a tight pair of very low mass companions to HD 65216 (projected separation of $255 \mathrm{AU}$ ), an early M companion to HD 177830 (projected separation of 97 AU), and we resolved the previously known companion to HD 196050 into a close pair of M dwarfs. Our data additionally confirm the bound nature of the companions to HD 142, HD 16141, and HD 46375. Among control stars, we detected true companions to HD 7895, HD 24331, HD 31412, HD 40397, HD 43834, HD 70923, HD 78351, HD 104263, HD 129642, HD 154682, and HD 223913, and likely bound companions to HD 82241 and HD 134180. Most of these objects are M dwarfs and have projected separations between 7 and 505 AU.
\end{abstract}

Key words. techniques: high angular resolution - stars: binaries: visual - stars: low-mass, brown dwarfs - stars: planetary systems

\section{Introduction}

Searches for planetary companions outside the Solar System have led to the discovery of more than 200 planets, most detected by the Doppler spectroscopy technique. Among the first objects unveiled, the three planets reported by Butler et al. (1997) were found to orbit the primary components of wide binaries. These discoveries were closely followed by the detection of $16 \mathrm{Cyg} b$ (Cochran et al. 1997), a planet orbiting the secondary component of a triple system. The existence of planets in wide binaries and multiple stars was thus supported by observational evidence very early. The discovery of Gl $86 \mathrm{~b}$ a few years later (Queloz et al. 2000), showed that Jovian planets can also form and survive in much closer binaries, raising the possibility that planets might be common in double and multiple star systems.

^ Based on observations collected at the ESO VLT Yepun telescope, proposals 70.C-0557, 71.C-0125, 73.C-0124, 74.C-0048, 75.C-0069, and 76.C-0057.

$\star \star$ Tables 1, 2, and Figs. 3, 4 are only available in electronic form at http://www . aanda.org
Planets residing in binaries and multiple stars may possess some distinctive characteristics when compared to planets in orbit around single stars. Zucker \& Mazeh (2002) were the first to point out that planets found in binaries may follow a different period-mass correlation than planets orbiting single stars. Eggenberger et al. (2004b) pursued the analysis, showing that planets with orbital periods shorter than about 40 days and found in binaries may also follow a different period-eccentricity distribution. Although very interesting, these findings are not yet on a firm footing, as they suffer from two major shortcomings. First they rely on a small sample of planets found in binaries, hence their high sensitivity to the addition of new candidates; second, planet-host stars without binary companions were assumed to be single, though the presence of stellar companions had never been probed systematically. Conducting dedicated searches for planets in binaries on the one hand, and for stellar companions to the known planet-host stars on the other, constitute therefore two prerequisite steps in more comprehensive studies aimed at investigating how companion stars affect planetary properties. 
The presence of a close stellar companion is likely to influence planet formation. Two main mechanisms have been proposed for the formation of Jovian planets: the accretion of solid planetesimals followed by gas capture (the core accretion model; see e.g. Lissauer \& Stevenson (2007) for a review and references), or the fragmentation of a gravitationally unstable disk (the disk instability model; see e.g. Durisen et al. (2007) for a review and references). Regardless of the exact formation process, tidal perturbations from a close binary companion will truncate and heat any circumstellar protoplanetary disk (Artymowicz \& Lubow 1994; Nelson 2000; Pichardo et al. 2005), presumably affecting planet formation. Theoretical predictions as to how exactly planet formation will be affected are still debated. According to Nelson (2000), giant planet formation is inhibited in equal-mass binaries with a separation of $50 \mathrm{AU}$ whatever the formation mechanism, whereas Boss (2006) claims that giant planets are able to form in binaries with periastrons as small as 25 AU. On the other hand, Mayer et al. (2005) showed that the protoplanetary disk mass has a strong impact on the final results and that the two possible formation mechanisms yield different predictions as to the occurrence of giant planets formed in lowmass disks. Planets in binaries might then provide a unique data set to test these theoretical predictions and to possibly identify the main formation mechanism for giant planets.

Quantifying the frequency of planets in double and multiple stars is not as straightforward as it seems because planet searches based on Doppler spectroscopy are biased against close binaries. Double stars closer than 2-6" are indeed difficult targets for radial-velocity measurements, as the two components simultaneously contribute to the recorded flux. This not only introduces additional possibilities for spurious velocity variations, but it also makes it much more difficult to precisely extract the radial velocity of one individual component. Close binaries have consequently been left out of the major Doppler surveys and current data only provide sparse information on their suitability for planetary systems.

Recognizing the importance and the interest of including close binary stars in extrasolar planet studies, we have been investigating the impact of stellar duplicity on planet occurrence and properties for a few years. In this series, we will present and discuss the results from this dedicated investigation, which has two main facets. One of our goals is to directly quantify the occurrence of giant planets in binaries with very different separations, from wide common proper motion pairs down to spectroscopic systems. Although close binaries are not well-suited targets for radial-velocity planet searches, dedicated reduction techniques based on two-dimensional correlation have recently been developed to simultaneously extract the velocity of each component (Zucker et al. 2003; Konacki 2005). In many instances, the precision achieved by these techniques is sufficient to search for Jovian planets around the primary star, meaning that Doppler surveys for circumprimary giant planets in close binaries are feasible. By combining the results from our "classical" radial-velocity planet searches with those from our dedicated survey for giant planets in single-lined spectroscopic binaries (Eggenberger et al. 2003, 2007; Eggenberger \& Udry 2007), we should therefore be able to quantify the occurrence of giant planets in binaries as a function of the binary separation.

Another approach to the study of planets in binaries is to use direct imaging to trace out how stellar duplicity impacts on planet formation and evolution. For instance, if the presence of a close stellar companion hinders planet formation or drastically reduces the potential stability zones, then the frequency of planets in close binaries should be lower than the nominal frequency of planets orbiting single stars. Alternatively, if the presence of a close stellar companion stimulates planet formation one way or another, planets should be more common in close binaries than around single stars. Reversing these statements, studying the multiplicity of planet-host stars relative to that of stars without planetary companions may be a means of quantifying whether or not stellar duplicity has a negative impact on planet formation and/or evolution.

High-resolution imaging in the near infrared is an effective technique for detecting low-mass companions close to solar-type stars. Using the Keck adaptive optics, Luhman \& Jayawardhana (2002) were the first to conduct a systematic survey for companions to 25 planet-host stars. They identified 15 companion candidates, however all were probably background stars. Not long afterwards, combining high-resolution and wide-field imaging, Patience et al. (2002) found 3 true companions to their 11 planetbearing stars. On the basis of their small survey, Patience et al. (2002) concluded that the companion star fraction for planethost stars is not significantly different from that of field stars. However, enlarging the sample by taking into account the null result of Luhman \& Jayawardhana (2002), the difference between the two companion star fractions increases. With more than 150 planet-host stars known today, the companion star fraction of nearby stars with and without planets clearly merits reconsideration.

For some time, Mugrauer and coworkers have been searching for wide companions to a large number of planet-host stars, detecting several new systems (Mugrauer et al. 2004a,b, 2005b, 2006, 2007). On the other hand, using various catalogs and multiepoch images from the STScI Digitized Sky Survey, Raghavan et al. (2006) performed a systematic reconnaissance for stellar companions to the 131 planet-host stars known as of July 2005. This study revealed a few new systems and brought further support to the observed deficiency of stellar companions around planet-host stars. Yet, neither this study nor that by Patience et al. (2002) took into account the selection effects against close binaries in the samples of targets for Doppler planet searches, implying that the observed deficiency could be entirely due to these biases. Additionally, neither the survey by Mugrauer and coworkers nor the study by Raghavan et al. (2006) probed the innermost regions (separations below $100 \mathrm{AU}$ ) surrounding planet-bearing stars, leaving the most interesting and sensitive separation range largely unsurveyed. These innermost regions have been probed recently by Chauvin et al. (2006) for a sample of 26 planet-bearing stars, but mainly to characterize the nature (stellar or substellar) of additional companions revealed by the radial-velocity drift they induce on their parent star. As it is biased towards planet-bearing stars with potential stellar companions, this small sample is not well suited to study the companion star fraction for planet-host stars.

Since 2002 we have been conducting a large-scale systematic adaptive optics search for close stellar companions to nearby stars with and without planets (Eggenberger et al. 2004c; Udry et al. 2004; Eggenberger et al. 2007; Eggenberger \& Udry 2007). This program is aimed at characterizing and quantifying the major effects of stellar duplicity on planet occurrence and properties, with an emphasis as to whether or not the occurrence of giant planets is reduced in the presence of a close stellar companion. In order to access a large part of the celestial sphere, the main program was divided into two subprograms: a northern and a southern survey. In this first paper we report the observational results from our southern survey carried out with the NAOS-CONICA (NACO) facility at the European Southern Observatory (ESO) Very Large Telecope (VLT), Chile. 
The survey itself is presented in Sect. 2. Our observations and data reduction technique are described in Sects. 3 and 4, respectively. All the companion candidates detected on our images are listed in Sect. 5, where we discuss their likely origin as bound companions or unbound field stars. In Sect. 6 we summarize our observational results and briefly outline the next steps in the scientific interpretation of these new results. The statistical analysis aimed at investigating the global impact of stellar duplicity on planet occurrence is entirely deferred to the second paper in this series.

\section{The NACO survey}

\subsection{Sample}

As already mentioned, one limitation that prevents most imaging programs from drawing robust conclusions on the impact of stellar duplicity on planet occurrence, is the absence of a welldefined control sample for comparison. Previous studies used as reference the results from the multiplicity study of solar-type stars by Duquennoy \& Mayor (1991), but this is not entirely satisfactory for two reasons. First, Doppler planet searches suffer from noticeable selection effects against close binaries and these biases must be taken into account to obtain meaningful results. Second, statistical studies must compare the multiplicity among planet-host stars with the multiplicity among similar stars but without planetary companions, which is obviously a trickier issue to deal with. In an effort to be as rigorous as possible, we included in our NACO survey both planet-host stars and a control sub-sample of nearby field stars selected within our CORALIE planet search sample (Udry et al. 2000) and showing no obvious evidence for planetary companions from radial-velocity measurements. Proceeding in this way, we have at hand highprecision radial-velocity data which place constraints on the potential planet-bearing status of each control star, we match the target selection criteria for Doppler planet searches, and we minimize the corrections related to observational effects. Note that we additionally used the control stars as reference point spread function (PSF) stars to characterize the adaptive optics (AO) system performance and to identify PSF artifacts on each of our images.

Our NACO survey therefore relies on a sample of 57 planethost stars, together with 73 control stars carefully chosen so that they can be used both as comparison stars for the scientific analysis and as PSF reference stars in the data reduction process. Specifically, the two sub-samples were defined according to the following criteria. The planet-host star sub-sample comprises nearby stars: (i) known to harbor at least one planet candidate from the different radial-velocity planet search programs; (ii) observable from Paranal; (iii) not appearing in other published AO surveys. The control star sub-sample contains nearby stars: (i) belonging to our CORALIE planet search program (with the exception of two stars belonging to our ELODIE planet search program, Perrier et al. (2003)); (ii) with right ascension, declination, visual magnitude, color, and parallax as close as possible to the corresponding quantities of one of the planet-host stars; (ii) showing the least possible radial-velocity variations suggestive of the presence of a stellar or planetary companion on the basis of CORALIE and CORAVEL ${ }^{1}$ data; (iv) not listed as close

1 The two CORAVEL instruments (Baranne et al. 1979) were used extensively between 1977 and 1998 to monitor the radial velocity of more than 60000 nearby stars in both hemispheres. Although not precise enough to detect planets (typical precision of $0.3 \mathrm{~km} \mathrm{~s}^{-1}$ ), these data are very useful in characterizing long-term radial-velocity variations induced by stellar companions. visual binaries in the Hipparcos catalog (ESA 1997). The target list for each sub-sample along with further information on these targets (magnitude, distance, spectral type) are given in Tables 1 and 2 available in electronic form.

Note that the two sub-samples defined above are generic and multipurpose. For the statistical analysis, they will be refined and properly redefined according to the different issues we want to investigate. Here, we make out a few general comments. First, some planet-host stars appearing in other published AO surveys will be added to our final sample, thereby balancing the two sub-sample sizes for the final analysis. Second, our CORALIE radial-velocity data for control stars usually exclude the presence of short-period massive planets, but these data do not place strong constraints on the existence of less massive and/or longer period planets. Nonetheless, given the many stringent requirements for the selection of control stars, choosing them within the CORALIE planet search sample is the best compromise. Third, most of our targets are closer than $50 \mathrm{pc}$ (see Tables 1 and 2). By using a field of view of $13^{\prime \prime}$ (see next section), and for a typical target distance of $35 \mathrm{pc}$, we are sensitive to stellar companions with a projected separation between a few AU and 230 AU. Our survey thus probes the most interesting and sensitive separation range according to planet formation models.

\subsection{Observing strategy}

The survey observing strategy consisted of taking a first image of each of our targets (planet-host and control stars) in order to detect companion candidates. It is well known, however, that many such objects are in fact unrelated background (or possibly foreground) stars rather than true companions. Given the proximity of our targets, multiepoch astrometry is an effective method of discriminating between real companions that share the proper motion of the main target (assuming orbital motion is much smaller than proper motion) and background objects without significant proper motion over a few years. For relatively wide and bright objects $\left(\rho \gtrsim 10^{\prime \prime}, K \lesssim 14\right)$, a preexisting astrometric epoch may be found in the data from the Two Micron All Sky Survey (2MASS, Skrutskie et al. 2006) and only one NACO observation is needed. Nevertheless, because of the high angular resolution and the small field of view of NACO, we cannot rely on such preexisting data in a general way. As far as possible, our targets with companion candidates were thus observed twice during the survey, to check for common proper motion. For some candidates, our survey also includes two-band photometry, which is another means of invalidating physical companionship.

\section{Observations}

\subsection{Adaptive optics imaging with NACO}

Our systematic search for stellar companions to nearby southern stars was carried out with the NACO facility mounted at the Nasmyth focus of the ESO VLT Yepun telescope. NACO is made of NAOS, an adaptive optics system (Rousset et al. 2003) which delivers turbulence-corrected images to CONICA, a high-resolution imager and spectrograph sensitive from 1 to $5 \mu \mathrm{m}$ (Lenzen et al. 2003). NAOS is equipped with both visible and infrared Shack-Hartmann wave-front sensors that can be operated either in a $14 \times 14$ array configuration with 144 active subapertures or in a $7 \times 7$ array configuration with 36 active subapertures. The wave-front correction is provided by a tip-tilt mirror and a deformable mirror with 185 piezo-stack actuators. The CONICA detector was originally an Aladdin2 InSb 
Table 3. Main characteristics of the three instrumental setups used for our survey.

\begin{tabular}{ccccc}
\hline \hline Filter & $\begin{array}{c}\lambda_{\mathrm{c}} \\
(\mu \mathrm{m})\end{array}$ & $\begin{array}{c}F W H M \\
(\mu \mathrm{m})\end{array}$ & Camera & $\begin{array}{c}\text { Field of view } \\
(\operatorname{arcsec} \times \operatorname{arcsec})\end{array}$ \\
\hline NB_1.26 & 1.257 & 0.014 & $\mathrm{~S} 13$ & $13 \times 13$ \\
NB_1.64 & 1.644 & 0.018 & $\mathrm{~S} 13$ & $13 \times 13$ \\
NB_2.17 & 2.166 & 0.023 & $\mathrm{~S} 27$ & $27 \times 27$ \\
\hline
\end{tabular}

$1024 \times 1024$ pixel array, but since May 2004 it has been replaced by an Aladdin3 InSb $1026 \times 1024$ array. CONICA houses four cameras optimized for observations between 1 and $2.5 \mu \mathrm{m}$, of which two (S13 and S27) were used for our survey.

Our targets were observed over the course of 13.5 nights divided into six individual runs (2002 December 18-20, 2003 May 28-29, 2004 June 21-23, 2004 November 6-7, 2005 May 6-7, and 2005 December 8-9). All observations were made through narrowband filters within the $J$ (NB_1.26), $H$ (NB_1.64), or $K$ band (NB_2.17), using the visible wavefront sensor and the targets themselves for wavefront sensing (see Table 3 for details on the different observational setups). For our brightest targets, an additional neutral density filter was used to reduce the flux (attenuation by a factor of 80) and avoid saturating the detector of CONICA. During our second observing run, we also used the N90C10 dichroic acting as a neutral density filter on the imaging channel, transmitting only $10 \%$ of the light to CONICA.

The observing strategy consisted of recording unsaturated images of our targets, observing in succession each star of a pair planet-host star - control star. Proceeding in this way, the data obtained for one target can be used as an estimate of the PSF for the other target, at least as long as no close companion is present around either of the two stars. For the survey, the $H$-band filter was preferred because it produces the optimum combination of spatial resolution and sensitivity, and because low-mass objects are relatively bright at these wavelengths. However, in the case of degraded AO correction (bad seeing, short correlation time), the $K$-band filter was used to maintain acceptable image quality. When a relatively bright companion was detected, a supplementary observation was sometimes (i.e. whenever possible with regard to atmospheric conditions and observing schedule) made in the $J$ band for color information.

Imaging was performed using the autojitter mode in which the telescope moves according to a random pattern in a box with a given size. For each target, multiple exposures were typically obtained at 7 different offset positions on the detector, the offsets being distributed within a box of $10^{\prime \prime}$ for the $K$-band filter and of $7^{\prime \prime}$ for the $H$ - and $J$-band filters. To observe a large number of stars with a high sensitivity (i.e high enough to detect stellar companions down to the bottom of the main sequence) the total integration time per target was set between 10 and 15 min. Detector integration times (DITs) ranged from 0.345 (the minimum value) to $30 \mathrm{~s}$ and were chosen so that the signal level stayed well within the linear range of the detector. For short DITs, individual images obtained on each position resulted from the mean of several subintegrations. All our observations are summarized in Table 6.

\subsection{Observing conditions and overall data quality}

Meteorological conditions prevailing during our different observing runs were varied. The second and third runs (2003 May and 2004 June) were performed under good atmospheric
Table 4. Mean plate scale and detector orientation for our different observing runs. The orientation angle gives the position of true North east of the vertical.

\begin{tabular}{cccc}
\hline \hline Epoch & Camera & $\begin{array}{c}\text { Plate scale } \\
\left(\text { mas pixel }^{-1}\right)\end{array}$ & $\begin{array}{c}\text { Orientation } \\
(\mathrm{deg})\end{array}$ \\
\hline 2002 Dec. & S13 & $13.16 \pm 0.03$ & $0.15 \pm 0.11$ \\
2002 Dec. & S27 & $26.92 \pm 0.10$ & $0.14 \pm 0.10$ \\
2003 May & S13 & $13.16 \pm 0.06$ & $0.23 \pm 0.17$ \\
2004 Jun. & S13 & $13.18 \pm 0.06$ & $-0.06 \pm 0.17$ \\
2004 Jun. & S27 & $26.91 \pm 0.14$ & $0.29 \pm 0.23$ \\
2004 Nov. & S13 & $13.19 \pm 0.06$ & $0.10 \pm 0.16$ \\
2004 Nov. & S27 & $26.89 \pm 0.14$ & $0.29 \pm 0.16$ \\
2005 May & S13 & $13.17 \pm 0.06$ & $0.06 \pm 0.15$ \\
2005 May & S27 & $26.88 \pm 0.13$ & $0.31 \pm 0.15$ \\
2005 Dec. & S13 & $13.16 \pm 0.08$ & $0.20 \pm 0.17$ \\
2005 Dec. & S27 & $26.99 \pm 0.11$ & $0.26 \pm 0.12$ \\
\hline
\end{tabular}

conditions (clear sky, seeing below 1.4"), the two December runs were performed under mediocre conditions (some thin cirrus, seeing below $2^{\prime \prime}$ ), while the two remaining runs (2004 November and 2005 May) were performed under poor conditions (cirrus, seeing up to $3^{\prime \prime}$ ). The quality of our data is characterized by Strehl ratios up to 0.46 , with median values of 0.06 in the $J$ band, 0.13 in the $H$ band, and 0.25 in the $K$ band. The typical angular resolution of our images (PSF median full-width at half maximum, FWHM) is $0.072^{\prime \prime}$ in $J, 0.068^{\prime \prime}$ in $H$, and $0.076^{\prime \prime}$ in $K$.

\subsection{Astrometric calibration}

To achieve good accuracy in the determination of the separation and position angle of the detected binaries, a precise knowledge of the plate scale and the on-sky orientation of CONICA are required. Our primary astrometric calibrator is the central region of the Trapezium Cluster in the Orion Nebula (McCaughrean \& Stauffer 1994). Astrometric calibration fields near this region were thus observed whenever possible (i.e. during our two December runs). Our other runs were calibrated using astrometric binaries listed in van Dessel \& Sinachopoulos (1993) (IDS 21506S5133), double stars from the Hipparcos catalog (ESA 1997) (HIP 108797 and HIP 66225), or binaries from the Sixth Catalog of Orbits of Visual Binary Stars ${ }^{2}$ (Hartkopf et al. 2001) (HD 121325, HD 197913, HD 131156). However, some discrepancies were observed in the results, indicating that systematics are involved in using different calibrators. Consequently, we used as a secondary calibrator only the binary IDS 21506S5133, which we recalibrated against Orion in December 2005. The plate scale and the orientation of CONICA for each epoch of observation are given in Table 4.

\section{Data reduction and analysis}

\subsection{Image processing}

We processed our NACO images with the ECLIPSE software (Devillard 1997). The raw images of each target were first median combined to produce a sky frame, which was subtracted from the raw data. The resulting images were then divided by a flat-field frame taken on the twilight sky and corrected for bad pixels. Poor-quality images and saturated frames were next identified and rejected, except when most frames in the sequence were saturated. In this case, all saturated images were kept, but the observation was flagged as not reliable for photometry.

\footnotetext{
${ }^{2}$ http://ad.usno.navy.mil/wds/orb6.html
} 
Good-quality frames were finally recentered with subpixel accuracy and stacked into a high signal-to-noise image.

We deconvolved our images using a custom-made program adapted from the method developed by Veran \& Rigaut (1998). This method takes into account the specificities of the NACO PSF by using as impulse response the PSF of the reference star related to the target. The object is modeled as a set of individual stars (point sources), whose accurate positions (astrometry) and amplitudes (photometry) are sought. The free parameters of the model are therefore the pixel coordinates and the flux of each star (the number of stars ranges from 1 to 3 and is considered as known a priori). These parameters are determined through a Levenberg-Marquardt minimization scheme in the Fourier domain, i.e. the model is compared to the actual data through an optical-transfer-function fit. Uncertainties in the free parameters can be derived either from the covariance matrix or using a Monte Carlo approach. Once computed, the best model is subtracted from the data, yielding a residual map in the space domain. This residual map is used to check a posteriori the possible existence of overlooked sources.

\subsection{Identification and characterization of companion candidates}

The high signal-to-noise image of each target was inspected visually to identify companion candidates (denoted CCs in figures and tables). The image was next deconvolved as explained in Sect. 4.1 to search for additional close point sources that might have escaped immediate detection. The astrometric and photometric parameters of the detected binaries were then extracted as described below. Since most of our observations were performed under non-photometric conditions, only relative photometry can be obtained from our images. Nonetheless, using additional data from the 2MASS and Hipparcos catalogs, we were able to derive individual apparent and absolute magnitudes. For bound systems, these absolute magnitudes were finally used to estimate the spectral type and, whenever possible, the mass of the companions.

\subsubsection{Astrometry}

The observed astrometric parameters (separation and position angle) of relatively bright companion candidates (signal-to-noise ratio $\geq 20-30$ ) were derived from the outputs of our deconvolution program and set on an absolute scale using the calibration given in Table 4. As to the uncertainty budget, it was often dominated by external uncertainties rather than by internal fitting errors. External uncertainties were estimated for each system by performing the analysis on the stacked images obtained at each offset position (generally 7 offset positions, hence 7 images), taking the $1 \sigma$ confidence interval of the resulting set of values. Astrometric parameters given in Table 6 were thus obtained from the high signal-to-noise images, but the quoted uncertainties are the maximum values between internal and external errors.

For the faintest objects, approximative astrometric parameters were derived assuming that they were located on their maximum-flux pixel. Although crude, this approach gives fairly good results, owing to the small plate scales of the two CONICA cameras and to the relatively large proper motions of our targets (see Tables 1 and 2). The quoted uncertainties are external errors for the companion candidates visible on several stacked images, or otherwise correspond to a 1-pixel precision.

\subsubsection{Photometry}

Magnitude differences of relatively high signal-to-noise companion candidates were obtained from the outputs of our deconvolution program. For wide binaries, however, this approach is prone to systematics, as our program does not take into account PSF variations related to angular anisoplanatism. Such variations are actually present in our data, as attested by the typical PSF radial elongation visible on several of our images. Magnitude differences were consequently also estimated via aperture photometry using the IRAF/PHOT task. Specifically, the flux of each point source was measured with various aperture radii, from 3 pixels $(0.6-1.2 F W H M)$ up to about 80 pixels (16-32 FWHM), while the local sky value was determined as the mode of the flux distribution in a 10-pixel (2-4 FWHM) wide annulus with an inner radius 1.4 times larger than the aperture radius. For a given companion candidate, the aperture radius finally used was the smallest radius yielding an approximately constant magnitude difference (with respect to the primary star). The magnitude differences quoted in Table 6 are those obtained with our deconvolution program for binaries closer than $2^{\prime \prime}$, via aperture photometry for companions located further than $5^{\prime \prime}$, or are the mean of these two values for the intermediate separation range of $2-5^{\prime \prime}$ where both methods yielded compatible results. As for astrometry, the photometric uncertainty budget was usually dominated by external uncertainties, which were estimated using the stacked images obtained at each offset position.

Magnitude differences of faint companion candidates were determined via aperture photometry, or roughly estimated from the two peak values. Uncertainties are given only for the objects visible on several of the stacked images. Generally speaking, relative photometry is uncertain for the faintest objects and is not meant to be used in the analysis. It is merely given as complementary information for identification.

Since all our targets are nearby solar-type stars, they are listed in the 2MASS catalog, which provides us with nearinfrared $J, H$, and $K_{\mathrm{s}}$ magnitudes. In principle, we can thus obtain individual apparent magnitudes by combining our relative photometry with the 2MASS data. In practice, however, there are two additional complications: (1) our photometry was performed in narrowband filters that are different from the 2MASS broadband filters; (2) although resolved in 2MASS, intermediate systems (angular separation between about 4 and 10") with bright secondaries do not possess reliable photometry even for the primary stars. As explained below, the first issue was solved by correcting our NACO magnitude differences to put them on the 2MASS photometric system. Whenever uncontaminated and unbiased, the 2MASS photometry was then combined to our corrected magnitude differences to derive individual apparent magnitudes. Note that depending on the pair considered, the 2MASS photometry was either attributed to the combined system or to the primary star. Absolute magnitudes were finally computed using the Hipparcos parallaxes, taking into account interstellar extinction whenever it was not completely negligible. For the latter purpose, we used the extinction model by Chen et al. (1998) and the total to selective absorption ratios quoted in Cox (2000). For the few primaries with contaminated 2MASS photometry, near-infrared absolute magnitudes were estimated from the visual absolute magnitude (larger magnitude difference and higher angular resolution in the $V$ band) using the standard intrinsic colors for main-sequence stars from Cox (2000).

We estimated differences in the relative photometry between the NACO narrowband and the 2MASS broadband photometric systems using spectra from the library by Pickles (1998) to 
represent our targets (F8-K7 dwarfs with solar metallicity), and spectra from Leggett et al. (2000), Leggett et al. (2001), Leggett et al. (2002), Geballe et al. (2002), Knapp et al. (2004), and Chiu et al. (2006) to represent the detected companions (M dwarfs, L dwarfs/brown dwarfs). Specifically, for each filter we tabulated the difference (and hence the correction to apply) between the two photometric systems as a function of the spectral types of the two components. For the companions, the link between absolute magnitude and spectral type was established through mean empirical $M_{J}, M_{H}$, and $M_{K_{\mathrm{s}}}$ vs spectral type relations constructed using data from Delfosse et al. (2000), Leggett et al. (2000), Dahn et al. (2002), and Vrba et al. (2004) (see these last two papers for similar relations for late-M, L and T dwarfs). In a general way, observations made within one of the NACO narrowband filters underestimate the magnitude difference in comparison with observations performed in one of the 2MASS broadband filters. The largest differences are found in the $J$ band, and amount to $-0.36 \mathrm{mag}$ for a system made of an F8 primary and an L9 secondary.

\subsubsection{Masses}

Very low mass stars $\left(0.1 \lesssim M \lesssim 0.6 M_{\odot}\right)$ follow tight nearinfrared mass-luminosity relations (Delfosse et al. 2000), well reproduced by evolutionary models such as those by Baraffe et al. (1998). Masses of M-dwarf companions can therefore reliably be estimated from their near-infrared absolute magnitudes (and, strictly speaking, from their age, but fortunately the latter is not crucial for stars older than $1 \mathrm{Gyr}$, which is the case of most of our targets). The masses of companions with absolute magnitudes $M_{J} \in[5.5,11], M_{H} \in[5,10]$, or $M_{K_{\mathrm{s}}} \in[4.5,9.5]$ where then first derived from the Delfosse et al. (2000) relations (converted into the 2MASS photometric system), and secondly derived from the Baraffe et al. (1998) models (converted into the 2MASS photometric system as well), taking into account the age estimate (see Table 5) or using an age of 5 Gyr when no estimation was available. The mass finally quoted in Table 5 is the mean value between these two derivations, which usually agreed within their error bars.

Empirical mass-luminosity relations do not extend to lower mass companions (late- $\mathrm{M}$ and $\mathrm{L}$ dwarfs/brown dwarfs). Besides, the complexity of L dwarf atmospheres still challenges the present-day models, which remain approximate (see e.g. the discussions in Chabrier et al. 2000; and Burrows et al. 2006). In any case, our own data for these objects are sparse (one epoch and one filter) and not very precise. Therefore, only approximate spectral types were estimated for the companions with absolute magnitudes fainter than the range covered by the relations given in Delfosse et al. (2000).

\section{Results}

\subsection{Detected sources and companionship status}

Our data reveal 95 companion candidates found in the vicinity of 33 of our targets. The measured binary parameters (angular separation, position angle and magnitude difference) for all these potential systems are given in Table 6 . As mentioned earlier, we rely primarily on multiepoch astrometry to discriminate between true companions and unrelated objects. Astrometric parameters of bound systems are indeed not expected to vary much over the survey time span, except for some orbital motion in the closest systems. Alternatively, astrometric parameters of background objects without significant proper motion should vary according to the proper and parallactic motions of the primaries.

\subsubsection{Companion candidates with multiepoch observations}

Given the relatively large proper motions of our targets and the high angular resolution of NACO, two observations spanning a year or more can usually distinguish true companions from background stars with a reasonable confidence level (see Fig. 1 for examples). Such observations were obtained for 39 of our candidates. We additionally derived the companionship status of 22 other candidates by combining our one-epoch NACO observations with preexisting data from the literature (usually from the 2MASS catalog). For each of these 61 potential systems with multiepoch observations we then plotted the evolution of angular separation and position angle versus time, considering both the bound and unbound hypotheses as illustrated in Fig. 1. On the basis of these diagrams, we used the $\chi^{2}$ test to compute the probability for a given candidate to be bound or unbound. These results were finally used to classify our 61 companion candidates with multiepoch observations into five groups:

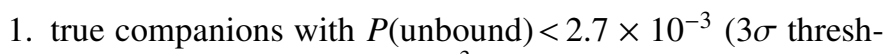
old) \& $P$ (bound $)>2.7 \times 10^{-3}$, or with $P($ unbound $)<2.7 \times$ $10^{-3} \&$ presence of a radial-velocity drift;

2. likely bound companions with $P$ (unbound $)<2.7 \times 10^{-3}$;

3. unbound companions with $P($ bound $)<2.7 \times 10^{-3} \quad \&$ $P($ unbound $) \geq 2.7 \times 10^{-3}$;

4. likely unbound companions with $P$ (bound $)<4.55 \times 10^{-2}(2 \sigma$ threshold) \& $P$ (unbound $) \geq 2.7 \times 10^{-3}$;

5. remaining candidates with inconclusive results.

Whenever available, our near-infrared multiband photometry was additionally used to check the correspondence of the companion candidates' colors with those expected for late-type dwarfs. Eighteen potential companions have unsaturated NACO photometry with an acceptable precision in at least two bands and three additional candidates have an uncontaminated photometry in the 2MASS catalog. Assuming that these 21 objects are located at the heliocentric distances of their respective primaries, we can place them on near-infrared color-magnitude diagrams such as those depicted in Fig. 2. To quantify the agreement of a companion's colors with those expected for main-sequence stars, we again used the $\chi^{2}$ test. Specifically, for each companion candidate we computed the difference in color (i.e. along the $x$ axis) between the observed point and the middle of the empirical sequence represented by the grey data points in Fig. 2 . At the $2 \sigma$ level, only the companion candidates to HD 162020 (CC1) (in the $H-K_{\mathrm{s}}$ diagram) and to HD 171587 (CC2) stand out as unrelated objects. This result is fully compatible with our astrometric data for these two objects (see Fig. 1). According to Fig. 2, the companion candidates to HD 74385 (CC1), HD 154682 (CC2), and to HD 168746 (CC9) look possibly unbound as well, but not with a very high confidence level $(1.8,1.9$, and $1.4 \sigma)$. Our astrometry shows that HD 74385 (CC1) and HD 154682 (CC2) are indeed background stars, while our astrometric results for HD 168746 (CC9) are inconclusive. The colors of the remaining 16 candidates are compatible with those of late-type dwarfs. Since red dwarfs constitute the bulk of the stellar population in the field, and since the M-dwarf sequence is almost vertical in near-infrared color-magnitude diagrams, this does not necessarily imply that all these candidates are true companions. Nonetheless, 14 out of these 16 objects also have a bound or likely bound status according to our multiepoch astrometry and are therefore bona fide companions. 


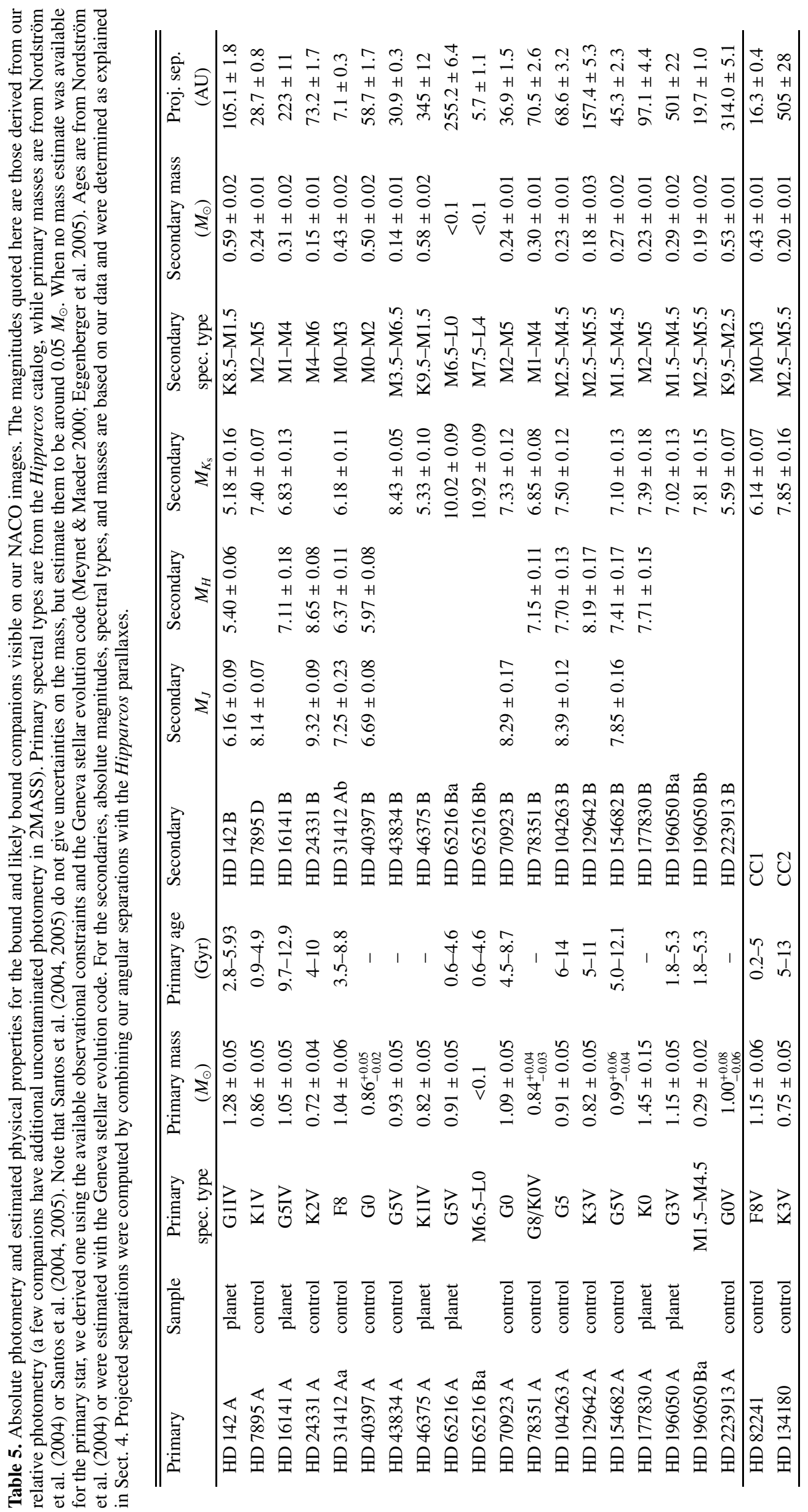



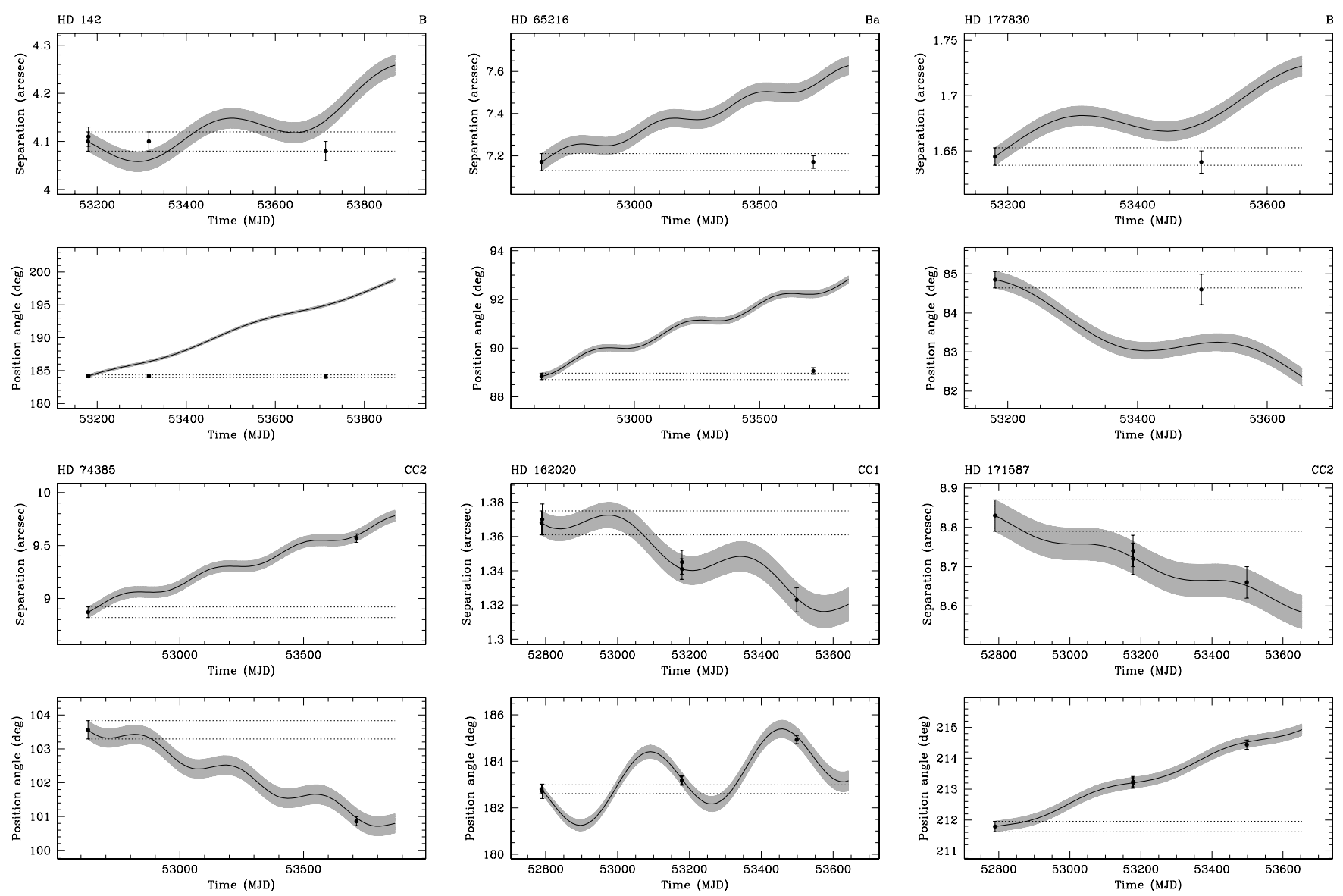

Fig. 1. Examples of multiepoch astrometry for three true companions (two upper lines) and for three companion candidates which are background stars (two lower lines). For each companion candidate, the upper panel shows the evolution of angular separation versus time and the lower panel shows the evolution of position angle versus time. Solid lines take into account the primary proper and parallactic motions, and thus depict the evolution expected for background objects with negligible proper motion. The grey zones represent the associated uncertainties, which comprise uncertainties on the parallax and on the proper motion of the primary star, along with the uncertainty on the first-epoch measurement.

To sum up, our multiband photometric results agree very well with the companionship status derived from our multiepoch astrometry, bringing further support to our previous conclusions. Among the candidates with multiepoch astrometry, we have 19 true companions (listed in Table 5 and designated according to the Washington Multiplicity Catalog nomenclature scheme, Hartkopf \& Mason 2004), 1 likely bound companion (HD 134180, also listed in Table 5 but still with its companion candidate designation), 27 unbound objects, 7 likely unbound objects, and 7 candidates with two-epoch data but inconclusive results. All our results and conclusions regarding companionship are summarized in Table 6 and are discussed on an individual case basis in the notes provided in the next two subsections. For the sake of conciseness, our astrometric results are represented graphically in Fig. 3 (available in electronic form) only for the true and likely bound companions. Our NACO images of true and likely bound systems are displayed in Fig. 4 (available in electronic form).

As mentioned earlier, the absolute near-infrared magnitudes derived from the combination of our data with the 2MASS photometry (or from the visual absolute magnitude in a few cases) were additionally used to derive approximate spectral types for all the true and likely bound companions, and masses for the brightest objects (spectral types earlier than $\sim \mathrm{M} 8$ ). These results are also given in the individual notes below and are summarized in Table 5. Among the true and likely bound companions with multiepoch data, we find 3 late-K-early-M stars, $15 \mathrm{M}$ dwarfs, and 2 late-M-early-L dwarfs.

\subsubsection{Companion candidates with only one observing epoch}

The companionship status of the 34 companion candidates with only one observing epoch is formally unknown. As discussed in the notes below, most of these candidates are likely unrelated objects, but a few may be true companions. To identify the most promising candidates in terms of physical companionship we computed likelihood of chance alignment on a per system basis. The probability of finding by chance one unrelated field star of brightness $m$ within an angular distance $\rho$ from a fixed point on the sky (i.e. from a specific target) is given by $P_{\mathrm{ca}}=N p(1-p)^{N-1}$, where $N=A(m)$ is the average surface density (per square degree) of stars with a magnitude $m \in[m-0.5, m+0.5]$, and $p=\pi\left(\rho^{2}-\rho_{\min }^{2}\right) / 3600^{2}$ is the probability of finding two stars separated by an angular distance $x \in\left[\rho_{\min }, \rho\right]$. The angular distance $\rho_{\min }$ is related to the detection limits and corresponds to the minimum angular separation at which a companion of magnitude $m$ can still be detected. Since our targets located near the galactic plane were often surrounded by several companion candidates with similar magnitudes, we sometimes computed the probability to have not only 1 , but 2 , 3 or more unrelated field stars with magnitude $m$ within a given angular radius. For all these calculations, we estimated average 

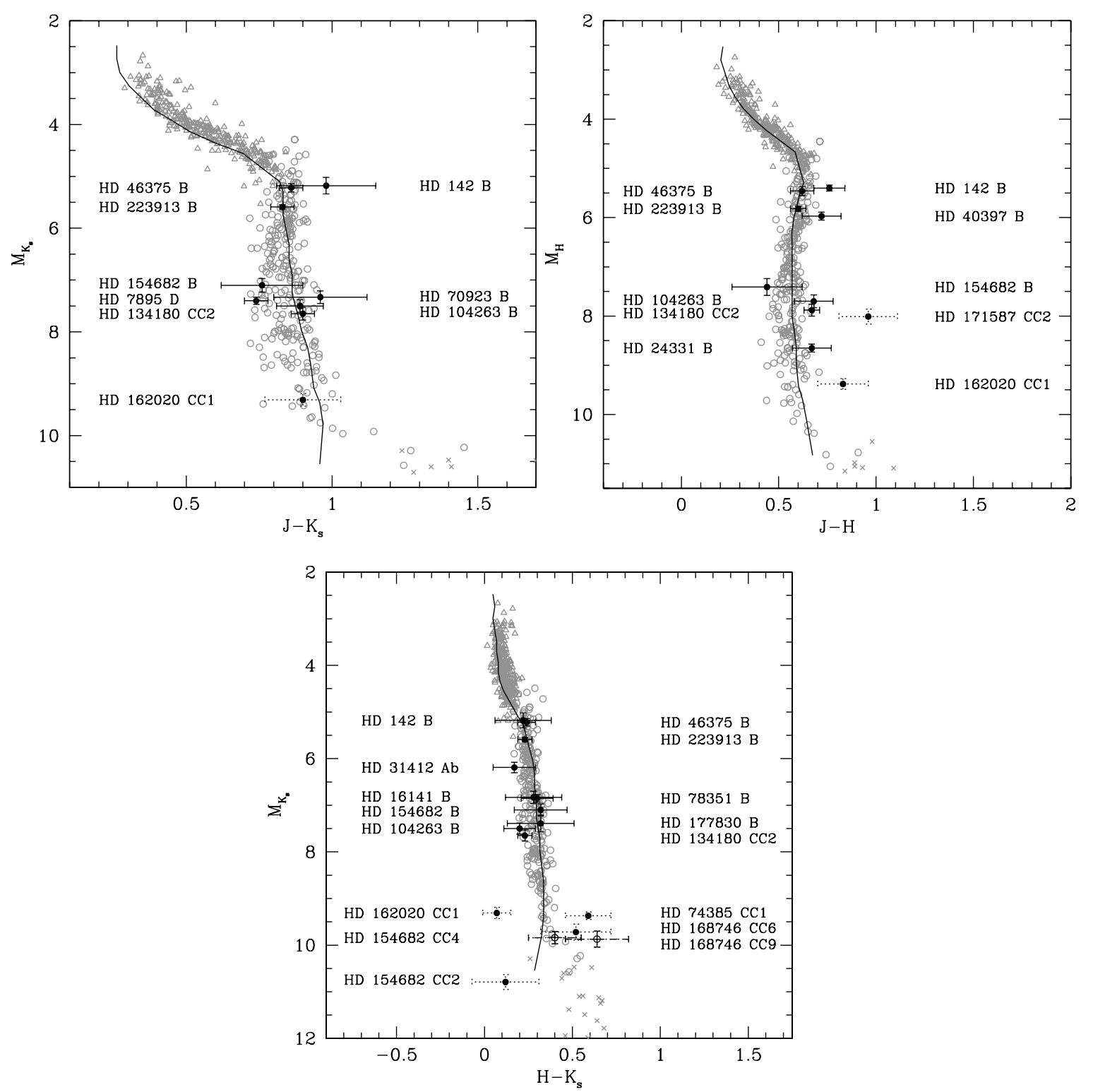

Fig. 2. Near-infrared color-magnitude diagrams for the 21 companion candidates with the most precise colors (uncertainties on apparent magnitudes less than 0.15). Dots and solid error bars denote true or likely bound companions, open circles and dashed error bars denote candidates with an unknown companionship status, while dots and dotted error bars denote unbound objects. These diagrams are based on our own photometric data, except for HD 46375, HD 134180, and HD 223913 for which we used the 2MASS photometry. For comparison, we also show main-sequence F7-K stars from Halbwachs et al. (2003) and Eggenberger et al. (2004a) (open triangles), M dwarfs from Delfosse and coworkers (open circles; Delfosse \& Forveille, private communication), and L dwarfs from Vrba et al. (2004). Known binaries have been removed from the samples of G, $\mathrm{K}$, and M stars. The solid line is a typical evolutionary model from Baraffe et al. (1998) (solar metallicity, $\mathrm{t}=5 \mathrm{Gyr}$, mixing length of one pressure scale height for masses below $0.7 M_{\odot}$, and of 1.9 scale height for higher masses). The reference photometric system is $2 \mathrm{MASS}$ and conversions were made whenever required.

surface densities using data from the 2MASS catalog, extrapolating a little the curves of $A(m)$ vs. $m$ for the companion candidates close to, or slightly below, the detection threshold of the 2MASS survey. A comparison with stellar counts derived from the Besançon models (Robin et al. 2003) shows that such an extrapolation is justified over the magnitude range covered by our faintest companion candidates.

The results of the calculations of likelihood of chance alignment are given in the individual notes in the next two subsections. In summary, only two companion candidates have a probability $P_{\mathrm{ca}}<1 \%$. These are the companion to HD 82241 $\left(P_{\mathrm{ca}}=4.8 \times 10^{-6}\right)$ and the outer companion to HD 149612
$\left(P_{\mathrm{ca}}=1.8 \times 10^{-4}\right)$. Although both objects may be true companions, we estimate that only the result for HD 82241 constitute enough evidence to add this object to our present list of likely bound objects. Strictly speaking, likelihood of chance alignment should be regarded in terms of ensemble statistics and there is a significant probability that a few additional candidates may be true companions as well. Yet, identifying these companions will require second-epoch observations. To obtain a robust multiplicity statistics for the closest systems, we are currently completing the second-epoch observations of all the companion candidates within $6.5^{\prime \prime}$ of our targets. 
Table 6. Summary of our observations, along with astrometric (angular separation, position angle) and photometric (magnitude difference in the NACO photometric system) parameters for the detected systems. Parentheses indicate saturation and hence unreliable photometry. The relative photometry of a few companions could not be determined because these objects were too close to the edges of our images. For each target, companion candidates are listed according to separation. True companions were also designated according to the Washington Multiplicity Catalog nomenclature scheme and this designation is given in the third column. The companionship status (as discussed in Sect. 5) is summarized in the last column.

\begin{tabular}{|c|c|c|c|c|c|c|c|c|}
\hline$\overline{\text { Target }}$ & Sample & Companion & $\begin{array}{l}\text { Epoch } \\
\text { (MJD) }\end{array}$ & Filter & $\begin{array}{c}\rho \\
(\operatorname{arcsec})\end{array}$ & $\begin{array}{c}\text { PA } \\
(\mathrm{deg})\end{array}$ & 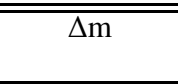 & Companionship \\
\hline \multirow{4}{*}{ HD 142} & planet & $\mathrm{CC} 1=\mathrm{B}$ & 53179.4118 & NB 1.64 & $4.10 \pm 0.02$ & $184.16 \pm 0.18$ & $3.09 \pm 0.05$ & bound \\
\hline & & & 53180.3242 & $\mathrm{NB} 1.26$ & $4.11 \pm 0.02$ & $184.16 \pm 0.18$ & $3.52 \pm 0.07$ & \\
\hline & & & 53316.1138 & NB_1.64 & $4.10 \pm 0.02$ & $184.18 \pm 0.18$ & $3.09 \pm 0.04$ & \\
\hline & & & 53713.0366 & NB_2.17 & $4.08 \pm 0.02$ & $184.13 \pm 0.29$ & $2.89 \pm 0.15$ & \\
\hline HD 2039 & planet & & 53180.3753 & NB_1.64 & & & & \\
\hline HD 3651 & planet & & 53317.0734 & NB_1.64 & & & & \\
\hline \multirow[t]{2}{*}{ HD 4203} & planet & & 53316.1536 & NB_1.64 & & & & \\
\hline & & & 53714.0034 & NB_2.17 & & & & \\
\hline HD 4208 & planet & & 52629.0400 & NB_2.17 & & & & \\
\hline HD 4308 & planet & & 53180.4019 & NB_1.64 & & & & \\
\hline \multirow[t]{3}{*}{ HD 4391} & control & & 53316.1322 & NB_1.64 & & & & \\
\hline & & & 53180.3504 & NB_1.26 & & & & \\
\hline & & & 53316.1322 & NB_1.64 & & & & \\
\hline HD 6107 & control & & 53180.4524 & NB_1.64 & & & & \\
\hline HD 6434 & planet & & 52628.0450 & NB_2.17 & & & & \\
\hline \multirow[t]{2}{*}{ HD 6715} & control & & 53317.0936 & NB_1.64 & & & & \\
\hline & & & 53317.1117 & NB 1.26 & & & & \\
\hline \multirow[t]{3}{*}{ HD 7895} & control & $\mathrm{CC} 1=\mathrm{D}$ & 53316.1849 & NB_1.64 & $1.036 \pm 0.005$ & $140.10 \pm 0.16$ & $(3.59 \pm 0.02)$ & bound \\
\hline & & & 53317.1304 & NB_1.26 & $1.037 \pm 0.005$ & $140.13 \pm 0.16$ & $3.71 \pm 0.02$ & \\
\hline & & & 53714.0279 & NB_2.17 & $1.038 \pm 0.004$ & $139.39 \pm 0.13$ & $3.45 \pm 0.01$ & \\
\hline HD 8638 & control & & 53178.4132 & NB_1.64 & & & & \\
\hline HD 8859 & control & & 52629.0620 & NB_2.17 & & & & \\
\hline HD 11112 & control & & 52628.0815 & NB_2.17 & & & & \\
\hline \multirow[t]{2}{*}{ HD 12042} & control & & 52627.0594 & NB_2.17 & & & & \\
\hline & & & 52629.1026 & NB_1.26 & & & & \\
\hline \multirow[t]{3}{*}{ HD 16141} & planet & $\mathrm{CC} 1=\mathrm{B}$ & 53317.1549 & NB_1.64 & $6.22 \pm 0.03$ & $187.12 \pm 0.17$ & $4.66 \pm 0.14$ & bound \\
\hline & & & 53317.1751 & $\mathrm{NB} \_1.26$ & $6.22 \pm 0.03$ & $187.13 \pm 0.17$ & $(4.81 \pm 0.20)$ & \\
\hline & & & 53714.0644 & NB_2.17 & $6.22 \pm 0.03$ & $186.77 \pm 0.14$ & $4.39 \pm 0.07$ & \\
\hline HD 18194 & control & $\mathrm{CC} 1$ & 53714.0889 & NB_2.17 & $10.21 \pm 0.04$ & $83.67 \pm 0.14$ & $8.51 \pm 0.21$ & likely unbound \\
\hline \multirow[t]{2}{*}{ HD 19034} & control & & 53317.2015 & NB_1.64 & & & & \\
\hline & & & 53317.2204 & NB_1.26 & & & & \\
\hline HD 20201 & control & & 52627.1011 & NB_2.17 & & & & \\
\hline HD 22610 & control & & 53713.0876 & NB_2.17 & & & & \\
\hline HD 23079 & planet & & 52627.0798 & NB_2.17 & & & & \\
\hline HD 24045 & control & & 52629.1661 & NB_2.17 & & & & \\
\hline \multirow[t]{3}{*}{ HD 24331} & control & $\mathrm{CC} 1=\mathrm{B}$ & 52627.1827 & NB_2.17 & $2.74 \pm 0.02$ & $22.85 \pm 0.10$ & $(4.13 \pm 0.06)$ & bound \\
\hline & & & 52627.1888 & NB_1.26 & $2.732 \pm 0.008$ & $22.89 \pm 0.18$ & $4.43 \pm 0.08$ & \\
\hline & & & 53316.2170 & NB_1.64 & $2.76 \pm 0.01$ & $22.61 \pm 0.16$ & $4.25 \pm 0.03$ & \\
\hline HD 25105 & control & & 53316.2567 & NB_1.64 & & & & \\
\hline HD 27894 & planet & & 53713.1094 & NB_2.17 & & & & \\
\hline HD 28185 & planet & & 52629.1458 & NB_2.17 & & & & \\
\hline HD 30177 & planet & & 53317.2620 & NB_1.64 & & & & \\
\hline HD 30278 & control & & 53317.2944 & NB_1.64 & & & & \\
\hline HD 30670 & control & & 53316.3254 & NB_1.64 & & & & \\
\hline \multirow[t]{3}{*}{ HD 31412} & control & $\mathrm{CC} 1=\mathrm{Ab}$ & 52628.1797 & NB_2.17 & $0.194 \pm 0.005$ & $69.26 \pm 0.30$ & $3.21 \pm 0.08$ & bound \\
\hline & & & 52628.1961 & NB_1.26 & $0.199 \pm 0.006$ & $68.56 \pm 0.44$ & $4.03 \pm 0.22$ & \\
\hline & & & 53714.1199 & NB_1.64 & $\sim 0.09^{3}$ & $\sim 95$ & - & \\
\hline \multirow[t]{2}{*}{ HD 33636} & planet & & 52628.1249 & NB_2.17 & & & & \\
\hline & & & 53714.1532 & NB_1.64 & & & & \\
\hline HD 38529 & planet & & 53713.2348 & NB_2.17 & & & & \\
\hline HD 38858 & control & & 53713.2556 & NB_2.17 & & & & \\
\hline \multirow[t]{2}{*}{ HD 39091} & planet & & 52629.1881 & NB_2.17 & & & & \\
\hline & & & 53713.1803 & NB_1.64 & & & & \\
\hline \multirow[t]{3}{*}{ HD 40397} & control & $\mathrm{CC} 1=\mathrm{B}$ & 52628.1454 & NB_2.17 & $2.57 \pm 0.01$ & $26.67 \pm 0.10$ & $(2.28 \pm 0.05)$ & bound \\
\hline & & & 52628.1643 & NB_1.26 & $2.565 \pm 0.006$ & $26.71 \pm 0.17$ & $2.72 \pm 0.06$ & \\
\hline & & & 53714.1777 & NB_1.64 & $2.48 \pm 0.02$ & $28.28 \pm 0.17$ & $2.40 \pm 0.04$ & \\
\hline HD 43834 & control & $\mathrm{CC} 1=\mathrm{B}$ & 52629.2094 & NB_2.17 & $3.02 \pm 0.01$ & $250.87 \pm 0.11$ & $4.97 \pm 0.05$ & bound \\
\hline
\end{tabular}


Table 6. continued.

\begin{tabular}{|c|c|c|c|c|c|c|c|c|}
\hline Target & Sample & Companion & $\begin{array}{l}\text { Epoch } \\
\text { (MJD) }\end{array}$ & Filter & $\begin{array}{c}\rho \\
(\operatorname{arcsec})\end{array}$ & $\begin{array}{c}\text { PA } \\
\text { (deg) }\end{array}$ & $\overline{\Delta m}$ & Companionship \\
\hline & & & 52629.2268 & NB_1.26 & $3.02 \pm 0.02$ & $251.14 \pm 0.27$ & $(5.41 \pm 0.20)$ & \\
\hline & & & 53713.2054 & NB_1.64 & $3.07 \pm 0.02$ & $252.31 \pm 0.20$ & $(5.24 \pm 0.13)$ & \\
\hline HD 45067 & control & & 52627.2717 & NB_2.17 & & & & \\
\hline \multirow[t]{2}{*}{ HD 46375} & planet & $\mathrm{CC} 1=\mathrm{B}$ & 53316.3543 & NB_2.17 & $10.29 \pm 0.05$ & $309.67 \pm 0.16$ & $1.89 \pm 0.10$ & bound \\
\hline & & & 53714.2499 & NB_2.17 & $10.34 \pm 0.04$ & $309.65 \pm 0.13$ & $1.90 \pm 0.06$ & \\
\hline HD 50554 & planet & & 52627.2517 & NB_2.17 & & & & \\
\hline HD 54359 & control & $\mathrm{CC} 1$ & 53714.2750 & NB_2.17 & $3.22 \pm 0.02$ & $328.45 \pm 0.15$ & $8.23 \pm 0.23$ & 1 epoch \\
\hline HD 55720 & control & & 52629.3234 & NB_2.17 & & & & \\
\hline HD 56560 & control & & 52628.2836 & NB_2.17 & & & & \\
\hline \multirow[t]{2}{*}{ HD 59468} & control & & 52629.2729 & NB_2.17 & & & & \\
\hline & & & 53714.3002 & NB_2.17 & & & & \\
\hline \multirow[t]{2}{*}{ HD 65216} & planet & $\mathrm{CC} 1=\mathrm{Ba}$ & 52629.2503 & NB_2.17 & $7.17 \pm 0.04$ & $88.84 \pm 0.13$ & $6.37 \pm 0.19$ & bound \\
\hline & & & 53714.3228 & NB_2.17 & $7.17 \pm 0.03$ & $89.06 \pm 0.14$ & $6.31 \pm 0.06$ & \\
\hline \multirow[t]{2}{*}{$\mathrm{HD} 65216 \mathrm{Ba}$} & & $\mathrm{CC} 2=\mathrm{Bb}$ & 52629.2503 & NB_2.17 & $0.19 \pm 0.04$ & $236 \pm 11$ & - & bound \\
\hline & & & 53714.3228 & NB_2.17 & $0.144 \pm 0.001$ & $224.14 \pm 0.70$ & $0.90 \pm 0.03$ & \\
\hline HD 67556 & control & & 53317.3211 & NB_1.64 & & & & \\
\hline HD 70642 & planet & & 53317.3418 & NB_1.64 & & & & \\
\hline \multirow[t]{4}{*}{ HD 70923} & control & $\mathrm{CC} 1=\mathrm{B}$ & 52628.3217 & NB_2.17 & $0.847 \pm 0.004$ & $25.30 \pm 0.22$ & $4.74 \pm 0.08$ & bound \\
\hline & & & 52629.3431 & NB_2.17 & $0.844 \pm 0.005$ & $25.27 \pm 0.28$ & $4.86 \pm 0.09$ & \\
\hline & & & 52629.3556 & NB_1.26 & $0.845 \pm 0.004$ & $25.51 \pm 0.26$ & $5.38 \pm 0.14$ & \\
\hline & & & 53713.3253 & NB_2.17 & $0.847 \pm 0.004$ & $21.00 \pm 0.23$ & $4.70 \pm 0.07$ & \\
\hline \multirow[t]{2}{*}{ HD 71835} & control & $\mathrm{CC} 1$ & 53713.2797 & NB_2.17 & $11.83 \pm 0.05$ & $10.92 \pm 0.16$ & $7.86 \pm 0.17$ & 1 epoch \\
\hline & & $\mathrm{CC} 2$ & 53713.2797 & NB_2.17 & $13.33 \pm 0.06$ & $280.15 \pm 0.13$ & $5.56 \pm 0.16$ & unbound \\
\hline HD 73256 & planet & & 52789.0094 & NB_1.64 & & & & \\
\hline HD 73524 & control & & 52789.9750 & NB_1.64 & & & & \\
\hline HD 73526 & planet & & 52788.9766 & NB_1.64 & & & & \\
\hline HD 74156 & planet & & 52628.3024 & NB_2.17 & & & & \\
\hline \multirow[t]{6}{*}{ HD 74385} & control & $\mathrm{CC} 1$ & 52628.3431 & NB_2.17 & $5.67 \pm 0.05$ & $323.29 \pm 0.13$ & $5.18 \pm 0.12$ & unbound \\
\hline & & & 52629.3789 & NB_1.26 & $5.68 \pm 0.02$ & $323.15 \pm 0.23$ & 6.3 & \\
\hline & & & 53496.9686 & NB_1.64 & $5.43 \pm 0.03$ & $330.24 \pm 0.15$ & $5.68 \pm 0.10$ & \\
\hline & & & 53714.3452 & NB_2.17 & $5.50 \pm 0.03$ & $331.56 \pm 0.24$ & $5.05 \pm 0.04$ & \\
\hline & & $\mathrm{CC} 2$ & 52628.3431 & NB_2.17 & $8.87 \pm 0.05$ & $103.56 \pm 0.27$ & 7.2 & unbound \\
\hline & & & 53714.3452 & NB_2.17 & $9.57 \pm 0.04$ & $100.86 \pm 0.13$ & $6.93 \pm 0.08$ & \\
\hline HD 75289 & planet & & 52789.9909 & NB_1.64 & & & & \\
\hline HD 76700 & planet & $\mathrm{CC} 1$ & 53713.3718 & NB_2.17 & $6.03 \pm 0.05$ & $238.47 \pm 0.38$ & $8.54 \pm 0.24$ & 1 epoch \\
\hline \multirow[t]{2}{*}{ HD 78351} & control & $\mathrm{CC} 1=\mathrm{B}$ & 52789.0367 & NB_1.64 & $1.827 \pm 0.008$ & $163.58 \pm 0.17$ & $3.36 \pm 0.02$ & bound \\
\hline & & & 53713.3006 & NB_2.17 & $1.806 \pm 0.007$ & $163.16 \pm 0.12$ & $3.10 \pm 0.02$ & \\
\hline HD 78538 & control & & 52627.3126 & NB_2.17 & & & & \\
\hline HD 78558 & control & & 52627.3608 & NB_2.17 & & & & \\
\hline HD 82241 & control & $\mathrm{CC} 1$ & 53713.3497 & NB_2.17 & $0.412 \pm 0.002$ & $280.79 \pm 0.12$ & $3.27 \pm 0.02$ & 1 epoch, likely bound \\
\hline HD 82943 & planet & & 52627.3366 & NB_2.17 & & & & \\
\hline \multirow[t]{2}{*}{ HD 83443} & planet & & 52628.3642 & NB_2.17 & & & & \\
\hline & & $\mathrm{CC} 1$ & 53714.3629 & NB_2.17 & $11.55 \pm 0.06$ & $111.69 \pm 0.22$ & $8.61 \pm 0.22$ & likely unbound \\
\hline HD 85390 & control & & 53496.9975 & NB_1.64 & & & & \\
\hline HD 92788 & planet & & 52787.9863 & NB_1.64 & & & & \\
\hline HD 98281 & control & & 52788.0105 & NB_1.64 & & & & \\
\hline HD 101581 & control & & 53497.0495 & NB_2.17 & & & & \\
\hline HD 102117 & planet & & 53497.0748 & NB_2.17 & & & & \\
\hline \multirow[t]{3}{*}{ HD 104263} & control & $\mathrm{CC} 1=\mathrm{B}$ & 53179.0123 & NB_1.64 & $1.603 \pm 0.007$ & $301.95 \pm 0.17$ & $4.27 \pm 0.06$ & bound \\
\hline & & & 53179.0372 & NB_1.26 & $1.606 \pm 0.008$ & $302.02 \pm 0.18$ & $4.57 \pm 0.05$ & \\
\hline & & & 53497.1047 & NB_2.17 & $1.598 \pm 0.008$ & $301.54 \pm 0.15$ & $4.12 \pm 0.05$ & \\
\hline HD 106116 & control & & 52788.0632 & NB_1.64 & & & & \\
\hline HD 106252 & planet & & 52788.0356 & NB_1.64 & & & & \\
\hline HD 107885 & control & & 52790.0245 & NB_1.64 & & & & \\
\hline HD 108147 & planet & & 52790.0077 & NB_1.64 & & & & \\
\hline HD 108523 & control & & 53497.1416 & NB_2.17 & & & & \\
\hline HD 108874 & planet & & 53179.0661 & NB_1.64 & & & & \\
\hline HD 111232 & planet & & 53178.0136 & NB_1.64 & & & & \\
\hline HD 114386 & planet & & 52790.0418 & NB_1.64 & & & & \\
\hline HD 114729 & planet & & 52790.0870 & NB_1.64 & & & & \\
\hline
\end{tabular}


Table 6. continued.

\begin{tabular}{|c|c|c|c|c|c|c|c|c|}
\hline Target & Sample & Companion & $\begin{array}{l}\text { Epoch } \\
\text { (MJD) }\end{array}$ & $\overline{\text { Filter }}$ & $\begin{array}{c}\rho \\
(\operatorname{arcsec})\end{array}$ & $\begin{array}{c}\text { PA } \\
\text { (deg) }\end{array}$ & $\overline{\Delta \mathrm{m}}$ & "Companionship \\
\hline HD 114747 & control & & 52790.0669 & NB_1.64 & & & & \\
\hline HD 114783 & planet & & 52789.0713 & NB_1.64 & & & & \\
\hline HD 115585 & control & & 53178.0423 & NB_1.64 & & & & \\
\hline HD 117105 & control & & 52790.1109 & NB_1.64 & & & & \\
\hline HD 121504 & planet & & 52789.1356 & NB_1.64 & & & & \\
\hline HD 123265 & control & & 52788.1386 & NB_1.64 & & & & \\
\hline HD 125455 & control & & 52789.0977 & NB_1.64 & & & & \\
\hline HD 125881 & control & & 53180.0378 & NB_1.64 & & & & \\
\hline HD 128311 & planet & & 52789.1147 & NB_1.64 & & & & \\
\hline \multirow[t]{6}{*}{ HD 129642} & control & $\mathrm{CC} 1=\mathrm{B}$ & 53180.0655 & NB_1.64 & $5.51 \pm 0.03$ & $342.42 \pm 0.18$ & $4.69 \pm 0.16$ & bound \\
\hline & & & 53497.1781 & NB_2.17 & $5.50 \pm 0.03$ & $342.42 \pm 0.19$ & $(4.33 \pm 0.06)$ & \\
\hline & & $\mathrm{CC} 2$ & 53180.0655 & NB_1.64 & $5.33 \pm 0.03$ & $58.61 \pm 0.26$ & 8.9 & unbound \\
\hline & & & 53497.1781 & NB_2.17 & $5.97 \pm 0.03$ & $58.51 \pm 0.29$ & $(9.03 \pm 0.20)$ & \\
\hline & & $\mathrm{CC} 3 \mathrm{ab}^{4}$ & 53497.1781 & NB_2.17 & $8.93 \pm 0.05$ & $94.35 \pm 0.19^{2}$ & $(7.76 \pm 0.18)$ & 1 epoch \\
\hline & & $\mathrm{CC} 4$ & 53497.1781 & NB_2.17 & $12.00 \pm 0.07$ & $131.87 \pm 0.23$ & $(7.63 \pm 0.34)$ & unbound \\
\hline HD 130322 & planet & & 52788.1760 & NB_1.64 & & & & \\
\hline \multirow[t]{2}{*}{ HD 134180} & control & CC1? & 53497.2107 & NB_2.17 & $0.90 \pm 0.02$ & $246.94 \pm 0.56$ & 6.5 & 1 epoch, real? \\
\hline & & $\mathrm{CC} 2$ & 53497.2107 & NB_2.17 & $13.59 \pm 0.07$ & $311.60 \pm 0.16$ & $3.51 \pm 0.11$ & likely bound \\
\hline HD 134987 & planet & & 52789.2370 & NB_1.64 & & & & \\
\hline HD 136118 & planet & & 52790.1339 & NB_1.64 & & & & \\
\hline HD 136257 & control & & 52790.1517 & NB_1.64 & & & & \\
\hline HD 141937 & planet & & 52789.2189 & NB_1.64 & & & & \\
\hline HD 142415 & planet & & 52789.1753 & NB_1.64 & & & & \\
\hline HD 143114 & control & & 53180.1229 & NB_1.64 & & & & \\
\hline HD 145518 & control & & 53180.1471 & NB_1.64 & & & & \\
\hline \multirow[t]{5}{*}{ HD 145666} & control & $\mathrm{CC} 1$ & 53498.2068 & NB_2.17 & $2.08 \pm 0.02$ & $169.25 \pm 0.54$ & $7.72 \pm 0.10$ & 1 epoch \\
\hline & & $\mathrm{CC} 2$ & 53498.2068 & NB_2.17 & $2.42 \pm 0.02$ & $58.29 \pm 0.24$ & 9.2 & 1 epoch \\
\hline & & $\mathrm{CC} 3$ & 53498.2068 & NB_2.17 & $3.32 \pm 0.02$ & $152.71 \pm 0.43$ & $8.77 \pm 0.20$ & 1 epoch \\
\hline & & $\mathrm{CC} 4$ & 53498.2068 & NB_2.17 & $7.74 \pm 0.05$ & $245.71 \pm 0.17$ & $5.90 \pm 0.10$ & unbound \\
\hline & & $\mathrm{CC} 5$ & 53498.2068 & NB_2.17 & $13.56 \pm 0.07$ & $286.87 \pm 0.22$ & $7.53 \pm 0.36$ & unbound \\
\hline HD 146775 & control & & 53497.2795 & NB_2.17 & & & & \\
\hline HD 147513 & planet & & 53178.0942 & NB_1.64 & & & & \\
\hline \multirow[t]{11}{*}{ HD 149606} & control & $\mathrm{CC} 1$ & 53179.0943 & NB_1.64 & $3.20 \pm 0.02$ & $345.51 \pm 0.37$ & 9.8 & unbound \\
\hline & & & 53498.2068 & NB_2.17 & $3.45 \pm 0.04$ & $351.18 \pm 0.65$ & 9.9 & \\
\hline & & $\mathrm{CC} 2$ & 53179.0943 & NB_1.64 & $3.28 \pm 0.02$ & $359.37 \pm 0.37$ & 9.3 & unbound \\
\hline & & & 53498.2068 & NB_2.17 & $3.59 \pm 0.04$ & $4.42 \pm 0.63$ & 9.3 & \\
\hline & & $\mathrm{CC} 3$ & 53179.0943 & NB_1.64 & $4.75 \pm 0.03$ & $83.84 \pm 0.28$ & 9.7 & unbound \\
\hline & & & 53498.2068 & NB_2.17 & $5.07 \pm 0.04$ & $80.23 \pm 0.46$ & 9.7 & \\
\hline & & $\mathrm{CC} 4$ & 53179.0943 & NB_1.64 & $6.20 \pm 0.03$ & $122.91 \pm 0.24$ & $8.28 \pm 0.19$ & unbound \\
\hline & & & 53179.1173 & NB_1.26 & $6.20 \pm 0.03$ & $123.01 \pm 0.34$ & 8.3 & \\
\hline & & & 53498.2068 & NB_2.17 & $6.26 \pm 0.04$ & $119.01 \pm 0.21$ & $8.42 \pm 0.17$ & \\
\hline & & $\mathrm{CC} 5$ & 53179.0943 & NB_1.64 & $6.99 \pm 0.04$ & $235.50 \pm 0.23$ & 9.4 & unbound \\
\hline & & & 53498.2068 & NB_2.17 & $6.55 \pm 0.05$ & $236.07 \pm 0.36$ & 9.9 & \\
\hline \multirow[t]{3}{*}{ HD 149612} & control & $\mathrm{CC} 1$ & 52789.2001 & NB_1.64 & $5.84 \pm 0.03$ & $146.72 \pm 0.21$ & $6.72 \pm 0.23$ & unbound \\
\hline & & & 53498.2297 & NB_2.17 & $5.65 \pm 0.03$ & $140.09 \pm 0.22$ & $6.48 \pm 0.10$ & \\
\hline & & $\mathrm{CC} 2$ & 53498.2297 & NB_2.17 & $7.85 \pm 0.04$ & $333.91 \pm 0.20$ & $6.30 \pm 0.09$ & 1 epoch \\
\hline HD 153713 & control & & 52790.2018 & NB_1.26 & & & & \\
\hline \multirow[t]{5}{*}{ HD 154682} & control & $\mathrm{CC} 1=\mathrm{B}$ & 53178.1162 & NB_1.64 & $0.989 \pm 0.005$ & $78.56 \pm 0.18$ & $4.41 \pm 0.11$ & bound \\
\hline & & & 53178.1368 & NB_1.26 & $0.991 \pm 0.005$ & $78.46 \pm 0.18$ & $4.53 \pm 0.12$ & \\
\hline & & & 53497.3233 & NB_2.17 & $0.981 \pm 0.005$ & $78.22 \pm 0.16$ & $4.15 \pm 0.06$ & \\
\hline & & $\mathrm{CC} 2$ & 53178.1162 & NB_1.64 & $3.48 \pm 0.02$ & $139.67 \pm 0.26$ & $7.86 \pm 0.15$ & unbound \\
\hline & & & 53178.1368 & NB_1.26 & $3.50 \pm 0.02$ & $139.34 \pm 0.26$ & 7.8 & \\
\hline
\end{tabular}

\subsection{Individual notes for the planet-host stars with companion candidates}

HD 142. A bright companion candidate is visible on our images 4.1" south of HD 142. This object (LHS 1021, hereafter called HD 142 B) is listed in the Washington Visual Double Star Catalog (WDS) and in Poveda et al. (1994), and was identified as a common proper motion companion by Raghavan et al. (2006). Our high-resolution astrometric data bring unequivocal support to the bound nature of the system (Fig. 1). Although uncertain, our multiband photometry for this companion points towards a
K8.5-M1.5 star with a mass of $0.59 \pm 0.02 M_{\odot}$ (the near-infrared absolute magnitudes of the primary were derived from the visual photometry).

HD 16141. HD 16141 has a known common proper motion companion 6.22" south (Mugrauer et al. 2005a). Our data bring further support to the bound status of this pair. The combination of our astrometric data with those from Mugrauer et al. (2005a) seems to show some hints of orbital motion. Assuming a circular pole-on orbit, the expected orbital motion over the 3-year time span is $0.38 \mathrm{deg}$, about half of what is observed. The colors of HD 16141 B are consistent with those of an M1-M4 dwarf with 
Table 6. continued.

\begin{tabular}{|c|c|c|c|c|c|c|c|c|}
\hline Target & Sample & Companion & $\begin{array}{l}\text { Epoch } \\
\text { (MJD) }\end{array}$ & $\overline{\text { Filter }}$ & $\begin{array}{c}\rho \\
(\operatorname{arcsec})\end{array}$ & $\begin{array}{c}\text { PA } \\
\text { (deg) }\end{array}$ & 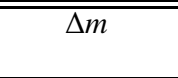 & "Companionship \\
\hline & & & 53497.3233 & NB_2.17 & $3.38 \pm 0.02$ & $139.53 \pm 0.22$ & $7.75 \pm 0.12$ & \\
\hline & & CC3 & 53497.3233 & NB_2.17 & $4.27 \pm 0.04$ & $113.03 \pm 0.52$ & 9.7 & 1 epoch \\
\hline & & $\mathrm{CC} 4$ & 53178.1162 & NB_1.64 & $4.76 \pm 0.03$ & $359.27 \pm 0.26$ & $7.21 \pm 0.13$ & inconclusive \\
\hline & & & 53178.1368 & NB_1.26 & $4.76 \pm 0.03$ & $359.27 \pm 0.26$ & 7.9 & \\
\hline & & & 53497.3233 & NB_2.17 & $4.81 \pm 0.03$ & $358.41 \pm 0.23$ & $6.83 \pm 0.07$ & \\
\hline & & $\mathrm{CC} 5$ & 53178.1162 & NB_1.64 & $5.00 \pm 0.03$ & $57.67 \pm 0.27$ & $7.93 \pm 0.31$ & likely unbound \\
\hline & & & 53178.1368 & NB_1.26 & $5.01 \pm 0.03$ & $57.75 \pm 0.27$ & 8.5 & \\
\hline & & & 53497.3233 & NB_2.17 & $4.97 \pm 0.03$ & $56.60 \pm 0.19$ & $7.61 \pm 0.10$ & \\
\hline & & CC6 & 53497.3233 & NB_2.17 & $5.98 \pm 0.03$ & $238.28 \pm 0.23$ & $8.83 \pm 0.23$ & 1 epoch \\
\hline & & $\mathrm{CC} 7$ & 53497.3233 & NB_2.17 & $6.35 \pm 0.05$ & $168.95 \pm 0.37$ & 10.0 & 1 epoch \\
\hline & & $\mathrm{CC} 8$ & 53497.3233 & NB_2.17 & $6.49 \pm 0.05$ & $52.79 \pm 0.34$ & 9.6 & 1 epoch \\
\hline & & CC9 & 53497.3233 & NB_2.17 & $6.73 \pm 0.04$ & $112.47 \pm 0.17$ & $8.94 \pm 0.18$ & 1 epoch \\
\hline & & CC10 & 53497.3233 & NB_2.17 & $9.99 \pm 0.06$ & $108.89 \pm 0.25$ & 9.9 & 1 epoch \\
\hline & & $\mathrm{CC} 11$ & 53497.3233 & NB_2.17 & $10.82 \pm 0.05$ & $244.08 \pm 0.17$ & $6.07 \pm 0.15$ & likely unbound \\
\hline & & $\mathrm{CC} 12$ & 53497.3233 & NB_2.17 & $19.68 \pm 0.10$ & $35.75 \pm 0.18$ & 6.4 & inconclusive \\
\hline \multirow{10}{*}{ HD 162020} & planet & $\mathrm{CC} 1$ & 52788.2350 & NB_1.64 & $1.368 \pm 0.007$ & $182.80 \pm 0.19$ & $5.19 \pm 0.06$ & unbound \\
\hline & & & 52790.1699 & NB_1.26 & $1.370 \pm 0.009$ & $182.71 \pm 0.31$ & $5.51 \pm 0.17$ & \\
\hline & & & 53179.1457 & NB_1.64 & $1.341 \pm 0.006$ & $183.18 \pm 0.18$ & $5.12 \pm 0.06$ & \\
\hline & & & 53179.1695 & NB_1.26 & $1.345 \pm 0.007$ & $183.18 \pm 0.21$ & $5.29 \pm 0.15$ & \\
\hline & & & 53498.2741 & NB_2.17 & $1.323 \pm 0.007$ & $184.93 \pm 0.17$ & $5.16 \pm 0.05$ & \\
\hline & & $\mathrm{CC} 2$ & 52788.2350 & NB_1.64 & $4.98 \pm 0.03$ & $228.37 \pm 0.20$ & 7.8 & inconclusive \\
\hline & & & 52790.1699 & NB_1.26 & $4.98 \pm 0.03$ & $228.37 \pm 0.34$ & $8.0 \pm 0.3$ & \\
\hline & & & 53179.1457 & NB_1.64 & $4.99 \pm 0.02$ & $228.70 \pm 0.19$ & $7.89 \pm 0.13$ & \\
\hline & & & 53179.1695 & NB_1.26 & $4.98 \pm 0.03$ & $228.60 \pm 0.21$ & $8.22 \pm 0.25$ & \\
\hline & & & 53498.2741 & NB_2.17 & $4.99 \pm 0.03$ & $229.06 \pm 0.19$ & $8.29 \pm 0.26$ & \\
\hline HD 162396 & control & & 52789.3128 & NB_1.64 & & & & \\
\hline \multirow[t]{24}{*}{ HD 168746} & planet & $\mathrm{CC} 1$ & 52789.2566 & NB_1.64 & $3.34 \pm 0.02$ & $142.97 \pm 0.26$ & $(8.6)$ & unbound \\
\hline & & & 53497.3605 & NB_2.17 & $3.27 \pm 0.02$ & $141.03 \pm 0.32$ & $8.66 \pm 0.22$ & \\
\hline & & $\mathrm{CC} 2$ & 52789.2566 & NB_1.64 & $3.60 \pm 0.02$ & $48.87 \pm 0.26$ & $(8.5)$ & unbound \\
\hline & & & 53497.3605 & NB_2.17 & $3.71 \pm 0.02$ & $47.33 \pm 0.21$ & $8.06 \pm 0.17$ & \\
\hline & & CC3 & 53497.3605 & NB_2.17 & $5.63 \pm 0.05$ & $243.00 \pm 0.40$ & 9.2 & 1 epoch \\
\hline & & $\mathrm{CC} 4$ & 53497.3605 & NB_2.17 & $5.87 \pm 0.05$ & $353.64 \pm 0.40$ & 9.0 & 1 epoch \\
\hline & & CC5 & 52789.2566 & NB_1.64 & $7.30 \pm 0.03$ & $107.57 \pm 0.26$ & (8.4) & unbound \\
\hline & & & 53497.3605 & NB_2.17 & $7.27 \pm 0.03$ & $106.45 \pm 0.18$ & $8.11 \pm 0.18$ & \\
\hline & & CC6 & 52789.2566 & NB_1.64 & $8.52 \pm 0.04$ & $289.41 \pm 0.17$ & $(6.92 \pm 0.15)$ & likely unbound \\
\hline & & & 53178.1666 & NB_1.64 & $8.56 \pm 0.04$ & $290.00 \pm 0.17$ & $7.04 \pm 0.14$ & \\
\hline & & & 53497.3605 & NB_2.17 & $8.51 \pm 0.04$ & $290.28 \pm 0.17$ & $6.54 \pm 0.14$ & \\
\hline & & $\mathrm{CC} 7$ & 53497.3605 & NB_2.17 & $8.84 \pm 0.06$ & $218.02 \pm 0.29$ & $8.12 \pm 0.20$ & 1 epoch \\
\hline & & $\mathrm{CC} 8$ & 52789.2566 & NB_1.64 & $9.00 \pm 0.05$ & $132.37 \pm 0.34$ & (7.8) & inconclusive \\
\hline & & & 53497.3605 & NB_2.17 & $8.94 \pm 0.04$ & $131.77 \pm 0.16$ & $7.17 \pm 0.18$ & \\
\hline & & CC9 & 53178.1666 & NB_1.64 & $10.44 \pm 0.05$ & $293.36 \pm 0.34$ & $7.31 \pm 0.10$ & inconclusive \\
\hline & & & 53497.3605 & NB_2.17 & $10.39 \pm 0.05$ & $293.63 \pm 0.19$ & $6.68 \pm 0.15$ & \\
\hline & & $\mathrm{CC} 10$ & 53497.3605 & NB_2.17 & $10.59 \pm 0.06$ & $337.19 \pm 0.25$ & 8.5 & 1 epoch \\
\hline & & $\mathrm{CC} 11$ & 53497.3605 & NB_2.17 & $12.22 \pm 0.06$ & $310.32 \pm 0.17$ & $7.86 \pm 0.31$ & 1 epoch \\
\hline & & $\mathrm{CC} 12$ & 53497.3605 & NB_2.17 & $12.80 \pm 0.07$ & $8.89 \pm 0.25$ & 8.1 & 1 epoch \\
\hline & & $\mathrm{CC} 13$ & 53497.3605 & NB_2.17 & $12.85 \pm 0.07$ & $333.50 \pm 0.16$ & $6.74 \pm 0.16$ & 1 epoch \\
\hline & & CC14 & 53497.3605 & NB_2.17 & $14.52 \pm 0.07$ & $338.17 \pm 0.15$ & $2.34 \pm 0.06$ & inconclusive \\
\hline & & CC15 & 53497.3605 & NB_2.17 & $19.55 \pm 0.10$ & $292.76 \pm 0.15$ & $5.67 \pm 0.16$ & inconclusive \\
\hline & & CC16 & 53497.3605 & NB_2.17 & $23.54 \pm 0.12$ & $308.76 \pm 0.16$ & $6.18 \pm 0.15$ & 1 epoch \\
\hline & & CC17 & 53497.3605 & NB_2.17 & $25.69 \pm 0.13$ & $311.72 \pm 0.16$ & $5.68 \pm 0.24$ & likely unbound \\
\hline HD 169830 & planet & & 52789.3298 & NB_1.64 & & & & \\
\hline HD 170493 & control & & 53180.1757 & NB_1.64 & & & & \\
\hline HD 171587 & control & $\mathrm{CC} 1$ & 52789.2774 & NB_1.64 & $3.93 \pm 0.02$ & $140.47 \pm 0.26$ & 8.8 & unbound \\
\hline & & & 53178.2026 & NB_1.64 & $3.69 \pm 0.02$ & $139.84 \pm 0.21$ & $8.51 \pm 0.20$ & \\
\hline & & & 53178.2321 & NB_1.26 & $3.70 \pm 0.03$ & $140.12 \pm 0.34$ & - & \\
\hline
\end{tabular}

a mass of $0.31 \pm 0.02 M_{\odot}$ (the near-infrared absolute magnitudes of the primary were derived from the visual photometry).

HD 46375. HD 46375 has a bright companion about 10" away (WDS, 2MASS). Mugrauer et al. (2006) and Raghavan et al. (2006) have shown that this system is physically bound. The one-year time span between our two NACO measurements is not sufficient to unambiguously distinguish between a true companion and an unrelated object. We therefore checked for common proper motion by combining our two astrometric data with the earlier 2MASS measurement. Doing so, we reach the same conclusion as Mugrauer et al. (2006) and Raghavan et al. (2006). The mean mass deduced from the 2MASS photometry and from our $K$-band observations is $0.58 \pm 0.02 M_{\odot}$. The 2 MASS colors of HD $46375 \mathrm{~B}$ are compatible with a late-K-early-M spectral type. 
Table 6. continued.

\begin{tabular}{|c|c|c|c|c|c|c|c|c|}
\hline Target & Sample & Companion & $\begin{array}{l}\text { Epoch } \\
\text { (MJD) }\end{array}$ & $\overline{\text { Filter }}$ & $\begin{array}{c}\rho \\
(\operatorname{arcsec})\end{array}$ & $\begin{array}{c}\text { PA } \\
\text { (deg) }\end{array}$ & $\overline{\Delta \Delta m}$ & "Companionship \\
\hline & & & 53498.2978 & NB_2.17 & $3.47 \pm 0.02$ & $139.71 \pm 0.21$ & $(8.73 \pm 0.17)$ & \\
\hline & & $\mathrm{CC} 2$ & 52789.2774 & NB 1.64 & $8.83 \pm 0.04$ & $211.79 \pm 0.17$ & $4.29 \pm 0.11$ & unbound \\
\hline & & & 53178.2026 & NB 1.64 & $8.72 \pm 0.04$ & $213.24 \pm 0.17$ & $4.22 \pm 0.10$ & \\
\hline & & & 53178.2321 & NB_1.26 & $8.74 \pm 0.04$ & $213.20 \pm 0.17$ & $4.79 \pm 0.11$ & \\
\hline & & & 53498.2978 & NB_2.17 & $8.66 \pm 0.04$ & $214.44 \pm 0.15$ & $(3.93 \pm 0.08)$ & \\
\hline & & $\mathrm{CC} 3$ & 53178.2321 & NB_1.26 & $13.54 \pm 0.06$ & $232.80 \pm 0.17$ & - & likely unbound \\
\hline & & & 53498.2978 & NB_ 2.17 & $13.51 \pm 0.07$ & $233.46 \pm 0.16$ & $(3.86 \pm 0.08)$ & \\
\hline & & $\mathrm{CC} 4$ & 53498.2978 & NB_ 2.17 & $13.56 \pm 0.08$ & $202.55 \pm 0.25$ & $(8.4)$ & 1 epoch \\
\hline & & CC5 & 53498.2978 & NB_2.17 & $14.50 \pm 0.08$ & $153.74 \pm 0.25$ & $(8.0)$ & 1 epoch \\
\hline & & CC6 & 53498.2978 & NB_2.17 & $14.61 \pm 0.08$ & $212.82 \pm 0.25$ & (8.3) & 1 epoch \\
\hline & & $\mathrm{CC} 7$ & 53498.2978 & NB_2.17 & $16.38 \pm 0.09$ & $144.89 \pm 0.18$ & (7.4) & unbound \\
\hline & & $\mathrm{CC} 8$ & 53498.2978 & NB_2.17 & $17.00 \pm 0.08$ & $304.87 \pm 0.15$ & $(4.63 \pm 0.10)$ & unbound \\
\hline & & CC9 & 53498.2978 & NB_2.17 & $18.44 \pm 0.09$ & $209.88 \pm 0.16$ & $(6.15 \pm 0.17)$ & unbound \\
\hline & & $\mathrm{CC} 10$ & 53498.2978 & NB_ 2.17 & $21.03 \pm 0.11$ & $167.96 \pm 0.18$ & - & unbound \\
\hline & & $\mathrm{CC} 11$ & 53498.2978 & NB_2.17 & $21.05 \pm 0.10$ & $220.81 \pm 0.16$ & $(6.00 \pm 0.17)$ & unbound \\
\hline & & $\mathrm{CC} 12$ & 53498.2978 & NB_2.17 & $21.80 \pm 0.11$ & $235.30 \pm 0.15$ & $(4.09 \pm 0.11)$ & unbound \\
\hline \multirow[t]{2}{*}{ HD 177830} & planet & $\mathrm{CC} 1=\mathrm{B}$ & 53180.1990 & NB_1.64 & $1.645 \pm 0.008$ & $84.85 \pm 0.21$ & $6.61 \pm 0.10$ & bound \\
\hline & & & 53498.3368 & NB_2.17 & $1.64 \pm 0.01$ & $84.60 \pm 0.39$ & $6.38 \pm 0.21$ & \\
\hline HD 179949 & planet & & 53179.1993 & NB_1.64 & & & & \\
\hline HD 184509 & control & & 53179.2266 & NB_1.64 & & & & \\
\hline HD 189567 & control & & 53498.3604 & NB_2.17 & & & & \\
\hline HD 192417 & control & & 53178.2630 & NB_1.64 & & & & \\
\hline \multirow[t]{2}{*}{ HD 196050} & planet & & 53178.2838 & NB_1.64 & & & & \\
\hline & & $\mathrm{CC} 1=\mathrm{Ba}^{5}$ & 53498.3838 & NB_2.17 & $10.68 \pm 0.05$ & $174.77 \pm 0.16$ & $3.84 \pm 0.06$ & bound \\
\hline HD $196050 \mathrm{Ba}$ & & $\mathrm{CC} 2=\mathrm{Bb}$ & 53498.3838 & NB_2.17 & $0.42 \pm 0.01$ & $165.43 \pm 0.30$ & $0.79 \pm 0.08$ & bound \\
\hline HD 202206 & planet & & 53180.2237 & NB_1.64 & & & & \\
\hline HD 204313 & control & $\mathrm{CC} 1$ & 53180.2475 & NB_1.64 & $6.22 \pm 0.03$ & $205.26 \pm 0.24$ & 9.1 & 1 epoch \\
\hline \multirow[t]{2}{*}{ HD 210277} & planet & & 52788.4308 & NB_1.64 & & & & \\
\hline & & & 52789.4079 & NB_1.26 & & & & \\
\hline HD 212168 & control & & 53180.2749 & NB 1.64 & & & & \\
\hline HD 213240 & planet & & 53178.3081 & NB_1.64 & & & & \\
\hline HD 213575 & control & & 52789.4296 & NB_1.26 & & & & \\
\hline HD 214759 & control & & 53271.1542 & NB_2.17 & & & & \\
\hline HD 215456 & control & & 53178.3582 & NB_1.64 & & & & \\
\hline HD 215657 & control & & 53178.3908 & NB_1.64 & & & & \\
\hline HD 216435 & planet & & 53178.3356 & NB_1.64 & & & & \\
\hline HD 216437 & planet & & 53180.3018 & NB_1.64 & & & & \\
\hline HD 216770 & planet & & 53271.0968 & NB_2.17 & & & & \\
\hline HD 220476 & control & & 53316.0493 & NB_1.64 & & & & \\
\hline HD 222582 & planet & & 53316.0221 & NB_1.64 & & & & \\
\hline HD 223913 & control & $\mathrm{CC} 1=\mathrm{B}$ & 53713.0622 & NB_2.17 & $12.14 \pm 0.05$ & $112.37 \pm 0.14$ & $2.36 \pm 0.06$ & bound \\
\hline \multirow[t]{6}{*}{ HD 330075} & planet & $\mathrm{CC} 1$ & 53180.0981 & NB_1.64 & $3.23 \pm 0.02$ & $347.33 \pm 0.24$ & $6.98 \pm 0.08$ & 1 epoch \\
\hline & & $\mathrm{CC} 2$ & 53180.0981 & $\mathrm{NB} 1.64$ & $4.25 \pm 0.02$ & $312.55 \pm 0.24$ & $8.11 \pm 0.22$ & 1 epoch \\
\hline & & $\mathrm{CC} 3$ & 53180.0981 & NB 1.64 & $6.80 \pm 0.03$ & $105.69 \pm 0.18$ & $8.09 \pm 0.16$ & 1 epoch \\
\hline & & $\mathrm{CC} 4$ & 53180.0981 & NB_1.64 & $7.78 \pm 0.04$ & $119.84 \pm 0.18$ & $7.29 \pm 0.12$ & 1 epoch \\
\hline & & CC5 & 53180.0981 & NB_1.64 & $8.81 \pm 0.04$ & $79.10 \pm 0.17$ & $7.34 \pm 0.21$ & 1 epoch \\
\hline & & CC6 & 53180.0981 & NB 1.64 & $8.93 \pm 0.04$ & $57.47 \pm 0.17$ & $6.17 \pm 0.10$ & unbound \\
\hline
\end{tabular}

${ }^{3}$ Due to the small separation, the results derived from our deconvolution method may be subject to systematic errors. The figures given here are therefore only indicative. ${ }^{4}$ Astrometry refers to CC3a, and photometry to CC $3 a b .{ }^{5}$ Photometry refers to the combined system Bab.

HD 65216. We detected a faint close binary about 7.2" east of HD 65216. This close pair is bound and shares the proper motion of HD 65216. HD 65216 is thus a new triple star system hosting a planet and the two newly detected companions will be denoted HD $65216 \mathrm{Ba}$ and HD $65216 \mathrm{Bb}$, respectively. From our $K$-band photometry we derive a spectral type between M6.5 and L0 for HD 65216 Ba and between M7.5 and L4 for HD 65216 Bb. These two companions are therefore very low mass stars at the bottom of the main sequence. These results are confirmed by an independent detection and analysis by Mugrauer et al. (2007).

HD 76700. We detected a faint point source 6 " south-west of HD 76700. The companionship status of this object is formally unknown as we only have one epoch and did not find additional data in the literature. From the galactic coordinates of HD 76700 $\left(l=282.5^{\circ}, b=-13.9^{\circ}\right)$ and from the apparent magnitude of this candidate $(K=15.23 \pm 0.24)$, we derive a likelihood of chance alignment $P_{\mathrm{ca}}=5.8 \%$.

HD 83443. Our images reveal a faint companion candidate $11.55^{\prime \prime}$ south-east of HD 83443. This object is listed in the 2MASS catalog but is close to the detection threshold and its photometry is of bad quality. Combining our single NACO observation with the previous 2MASS measurement, the system is classified as bound. Additional SofI images of HD 83443 are present in the ESO Science Archive Facility, since this star is also part of the survey by Mugrauer and coworkers for wide companions to planet-host stars (programs ID 70.C-0116 and 
073.C-0103). Using two epochs (2002 December 19 and 2004 July 2) together with the associated astrometric calibrations from Mugrauer et al. (2005b), our companion candidate is found to be likely unbound. Combining the SofI $H$-band photometry for the companion candidate with our NACO $K$-band photometry (converted into the 2MASS system), we reach the same conclusion. On that basis, and despite the contradictory result returned by the combination of our one-epoch NACO observation with the 2MASS data, we consider that the companion candidate to HD 83443 is likely unbound. A second-epoch NACO measurement would settle this point beyond doubt.

HD 162020. Our images reveal two point sources near HD 162020, the brightest one (CC1) about 1.3" in the south, the faintest one (CC2) about $5^{\prime \prime}$ in the south-west. CC1 is clearly a background star, as shown in Fig. 1. According to our astrometric data, $\mathrm{CC} 2$ is more likely unbound than bound, but the significance level of this result is quite low.

HD 168746. We detected 17 companion candidates near HD 168746. The galactic coordinates of this target $\left(l=19.0^{\circ}\right.$, $\left.b=+1.0^{\circ}\right)$ suggest that most of the objects visible on our images are background field stars rather than true companions. Among the candidates with two observing epochs, 3 are unbound, 2 are likely unbound, and 4 have inconclusive results but are more likely unrelated objects than true companions. The remaining candidates with only one observing epoch all have a likelihood of chance alignment greater than $6 \%$.

HD 177830. We discovered a companion candidate about 1.6" east of HD 177830. This companion shares the proper motion of HD 177830 (Fig. 1) and will consequently be denoted HD $177830 \mathrm{~B}$. The colors of HD $177830 \mathrm{~B}$ are consistent with those of an M2-M5 dwarf and a mass of $0.23 \pm 0.01 M_{\odot}$. Note that HD $177830 \mathrm{~A}$ is not a main-sequence star but a very evolved K0 subgiant.

HD 196050. HD 196050 has a known comoving companion about 10.7" south (Mugrauer et al. 2005a). Our high-resolution NACO data show that this companion is in fact not a single star but a close binary, whose two components will be termed $\mathrm{Ba}$ and $\mathrm{Bb}$. Computing the Bab photocenter and comparing it to the 2MASS data, the system is indeed comoving. This also brings some support to the bound nature of the close $\mathrm{Ba}-\mathrm{Bb}$ pair, which has only been resolved once. We estimate that $\mathrm{Ba}$ is an M1.5M4.5 dwarf with a mass of $0.29 \pm 0.02 M_{\odot}$, and $\mathrm{Bb}$ an M2.5M5.5 dwarf with a mass of $0.19 \pm 0.02 M_{\odot}$. HD 196050 is therefore actually a triple star system with a close (20 AU) pair of $\mathrm{M}$ dwarfs orbiting at $501 \mathrm{AU}$ from the G3 primary.

HD 330075. HD 330075 is surrounded by six companion candidates with angular projections between 3.23 and $8.93^{\prime \prime}$ and contrast ratios in the range 6.1-8.1 mag. The widest object (CC6) is listed in 2MASS and is clearly unbound. Given the galactic coordinates of HD $330075\left(l=330.8^{\circ}\right.$, $b=+3.4^{\circ}$ ), the remaining companion candidates are likely to be unrelated objects as well $\left(P_{\mathrm{ca}}(\mathrm{CC} 1)=8.8 \%, P_{\mathrm{ca}}(\mathrm{CC} 2)=27 \%\right.$, $P_{\mathrm{ca}}(\mathrm{CC} 3+\mathrm{CC} 2)=19 \%, \quad P_{\mathrm{ca}}(\mathrm{CC} 4+\mathrm{CC} 3+\mathrm{CC} 2)=11 \%, \quad P_{\mathrm{ca}}(>$ $\left.\left.3 \mathrm{CCs}, \mathrm{K} \sim 15.5, \rho \leq 8.81^{\prime \prime}\right)=9.9 \%\right)$.

\subsection{Individual notes for the control stars with companion candidates}

HD 7895. HD 7895 A forms a wide common proper motion pair with HD 7895 B, a K7 dwarf located about 28" away (Allen et al. 2000). A third component (HD 7895C) is listed in the WDS and was observed at 242.7" in 1907. Our NACO data resolve a fourth point source 1.04" south-east of HD $7895 \mathrm{~A}$. This object shares the proper motion of HD7895 A and its colors are compatible with those of an M2-M5 dwarf with a mass of $0.24 \pm 0.01 M_{\odot}$. Furthermore, our latest CORALIE measurements reveal a drift in the radial velocities, fully compatible with the variation expected from the orbital motion. This companion candidate is therefore physically related to HD $7895 \mathrm{~A}$ and will henceforth be denoted HD 7895 D. Regarding the companionship status of HD $7895 \mathrm{C}$, the closest object we found in the 2MASS catalog is 2MASS 01184607-0048029, whose position relative to HD $7895 \mathrm{~A}$ is roughly compatible with that expected for HD $7895 \mathrm{C}$ if it is a background star. On that basis, we consider that HD $7895 \mathrm{C}$ is likely to be an unrelated object. HD 7895 is thus a triple system consisting of a K1-early-M close pair orbited by a late-K wide companion.

HD 18194. Our images reveal a faint point source 10.21" east of HD 18194. Searching the literature for a second-epoch measurement, we found a source (SDSS J025510.05-075944.1) in the third release of the Sloan Digital Sky Survey (Abazajian et al. 2005) which roughly matches the expected position of our companion candidate, assuming it is a background star. The companion candidate to HD 18194 is thus probably an unrelated object. HD 24331. We detected a companion candidate 2.74 " north-east of HD 24331. This newly found object shares the proper motion of HD 24331 and will consequently be termed HD $24331 \mathrm{~B}$. We derive a projected separation of $73 \mathrm{AU}$ for this system and a mass of $0.15 \pm 0.01 M_{\odot}$ for the secondary. The colors of HD $24331 \mathrm{~B}$ point towards an M4-M6 spectral type.

HD 31412. Our images show a point source located at $0.20^{\prime \prime}$ from HD 31412 in 2002 December and at $\sim 0.09^{\prime \prime}$ in 2005 December. For this last epoch, the companion is clearly detected, but due to its closeness to the primary star we cannot derive reliable binary parameters with the deconvolution method described in Sect. 4.1. Yet, the significant motion observed between our two observing epochs is compatible with orbital motion but not with the motion expected for a background source. Moreover, the bound status of this companion candidate is supported by our ELODIE data, which show an increase in the radial velocity of about $1 \mathrm{~km} \mathrm{~s}^{-1}$ over 5 years. HD 31412 was in fact already known to have a wide companion (GJ9169 B, hereafter termed HD 31412 B) about 22" away. The separation and orientation of HD 31412 B with respect to HD 31412 A remained unchanged between 1960 (WDS) and 2000 (2MASS), indicating physical companionship (a $7^{\prime \prime}$ change in separation and a $15^{\circ}$ change in position angle would have been expected otherwise). HD 31412 is thus actually a triple system consisting of a close pair (HD 31412 Aa and the newly discovered HD 31412 Ab) orbited by the more distant HD 31412 B. HD $31412 \mathrm{Ab}$ is likely an early M dwarf with a mass of $0.43 \pm 0.02 M_{\odot}$.

HD 40397. HD 40397 has two companion candidates listed in the WDS: B (GJ 3376B) located at 4.1" in 1902, and C (HD 40374) located at 176.8" in 1898. HD 40397 B is visible on our images and is now located at $2.53^{\prime \prime}$ in the north-east. The apparent motion of B (both in our short-term data and over the 100-year time span) is not compatible with the expected motion of a background star but is fully consistent with orbital motion. The maximum radial-velocity acceleration expected from such a companion is about $10 \mathrm{~m} \mathrm{~s}^{-1} \mathrm{yr}^{-1}$, compatible with the $7.2 \mathrm{~m} \mathrm{~s}^{-1} \mathrm{yr}^{-1}$ drift observed in our CORALIE data. We estimate a mass of $0.50 \pm 0.02 M_{\odot}$ and an M0-M2 spectral type for HD 40397 B. Regarding HD 40374, its motion since 1898 shows that it is not physically related to HD 40397 . HD 40397 is thus a binary consisting of a G0 primary and an early M secondary.

HD 43834. We found a companion candidate 3.05" west of HD 43834. This object exhibits some motion relative to HD 43834, not enough to be consistent with that of a background 
star but fully compatible with orbital motion. This interpretation is further supported by the linear drift seen in our CORALIE data, also consistent with the orbital motion of $\mathrm{CC} 1$. This new companion candidate is thus a bona fide companion, and it will henceforth be called HD 43834B. It is likely an M3.5-M6.5 dwarf with a mass of $0.14 \pm 0.01 M_{\odot}$.

HD 54359. A faint point source is present on our images $3.22^{\prime \prime}$ north-west of HD 54359. We did not find any preexisting astrometric data for this object, whose companionship status remains unknown. Given the proximity of HD 54359 to the galactic plane $\left(b=-0.9^{\circ}\right)$ this companion candidate has a $2 \%$ probability of being an unrelated background star.

HD 70923. HD 70923 has a close companion (B $2527 \mathrm{~B}$, hereafter called HD 70923 B) observed at $0.2^{\prime \prime}$ in 1938 and at $0.1^{\prime \prime}$ in 1958 (WDS). This object is visible on our images and is now located $0.85^{\prime \prime}$ north of HD 70923. Our data show no significant variation in separation but a change of $4.5^{\circ}$ in position angle, fully compatible with the orbital motion expected for such a close pair. HD 70923 B is thus physically related to HD 70923 A. It is likely an M2-M5 dwarf with a mass of $0.24 \pm 0.01 M_{\odot}$. HD $70923 \mathrm{~A}$ has two additional companion candidates listed in the WDS: C located at $155.5^{\prime \prime}$ in 1919 , and D observed at 66.2" in 1908. HD 70923 C is listed in the Tycho Double Star Catalogue. These data show that the proper motion of HD 70923 C is radically different from that of HD 70923 A, and that its position in 1991.5 is that expected for a background star. The companionship status of D is not that obvious but we regard it as likely unbound on the basis that there is no object in the 2MASS catalog that approximately matches the position quoted in the WDS. HD 70923 is thus probably a regular binary. HD 71835. We found two point sources in the vicinity of HD 71835: CC1 at 11.8" in the north and CC2 at 13.3" in the west. CC2 is listed in 2MASS and is unbound. Our one-epoch observation does not allow us to conclude definitively on the companionship status of CC1. Nonetheless, HD 71835 is close to the galactic plane $\left(b=+1.0^{\circ}\right)$, making it likely $\left(P_{\mathrm{ca}}=24 \%\right.$, that CC1 is an unrelated field star as well.

HD 74385. HD 74385 has two companion candidates: CC1 about 5.5" in the north-west and CC2 about $9^{\prime \prime}$ in the east. Our data show that both these objects are background stars (see Fig. 1 for CC2). According to the WDS, HD 74385 has an additional companion candidate $45^{\prime \prime}$ away. The 2 MASS second-epoch data show that this source is bound and is probably an early $M$ dwarf, in agreement with the M1-M2 spectral type quoted in the literature.

HD 78351. Our data reveal a companion candidate $1.8^{\prime \prime}$ south of HD 78351. This object showed some motion between our two observing epochs, not in agreement with the motion expected for a background star but compatible with orbital motion. Its near-infrared colors are furthermore consistent with those of an M1-M4 dwarf with a mass of $0.30 \pm 0.01 M_{\odot}$. This companion candidate is thus physically bound to HD 78351 and it will be denoted HD $78351 \mathrm{~B}$.

HD 82241. We found a bright object $0.4^{\prime \prime}$ west of HD 82241 . Our single observation does not allow us to assess a definitive companionship status to this object but its proximity and brightness support a bound status $\left(P_{\mathrm{ca}}=4.8 \times 10^{-6}\right)$. If indeed bound, CC1 would be an M0-M3 dwarf with a mass of $0.43 \pm 0.01 M_{\odot}$. HD 82241 is in fact the secondary component of a wide pair $\left(108^{\prime \prime}\right)$, whose primary is HD 82207 . Both stars are listed in the Hipparcos catalog, and their relative position has not changed significantly since 1837 . This, and the fact that their respective proper motions are very similar, is an indication that the pair is physically bound (a 16" change in separation would have been expected otherwise). The Hipparcos data furthermore revealed an additional point source (component C) $0.68^{\prime \prime}$ away from HD 82207 but its relationship to HD 82207 (bound or unbound) is as yet unknown. HD 82241 is thus probably part of a multiple system and on the basis of the available data we classify CC1 as a likely bound object.

HD 104263. Our images show a point source $1.6^{\prime \prime}$ north-west of HD 104263. According to our astrometry and photometry, this object is physically related to HD 104263 and it will be denoted HD 104263 B. It is likely an M2.5-M4.5 dwarf with a mass of $0.23 \pm 0.01 M_{\odot}$. The small change in position angle that occurred between our two observing epochs is fully compatible with orbital motion.

HD 129642. HD 129642 is surrounded by 5 companion candidates, two of them forming a close pair. Our astrometric data show that the brightest candidate $(\mathrm{CC} 1)$ is a true companion, whereas $\mathrm{CC} 2$ and $\mathrm{CC} 4$ are unbound objects. The companionship status of the close pair CC3ab is unknown, but the galactic coordinates of HD $129642\left(l=321.0^{\circ}, b=+8.9^{\circ}\right)$ and the apparent magnitude of CC3ab $(K \sim 13.4)$ imply that CC3ab has a $8.3 \%$ probability of being an unrelated object. HD 129642 is thus probably a binary, made of a K3 primary and of an M2.5-M5.5 secondary with a mass of $0.18 \pm 0.03 M_{\odot}$.

HD 134180. A bright companion candidate is visible on our images 13.59" north-west of HD 134180. This object is also listed in the 2MASS catalog. Combining our one-epoch observation with the 2MASS data yields a likely bound status. Nonetheless, the change in separation angle seems quite large, calling for a confirmation of this status. As the 2MASS photometry of this object is uncontaminated, we can place it on a near-infrared color-magnitude diagram. This shows that its $J, H$, and $K_{\mathrm{s}}$ magnitudes are consistent with those of an M2.5-M5.5 dwarf. On the basis of these results we classify CC2 as a likely bound companion. Our images of HD 134180 show an additional speck of light at $0.90^{\prime \prime}$ in the south-west. With only one series of images, we cannot exclude that this tentative companion candidate is not actually a PSF artifact. Yet, HD 134180 is the only target of the night to have such a tentative companion candidate, whose position angle is furthermore not related to the telescope parallactic angle (see the discussion in Tokovinin et al. (2006)). Obviously, a second high-resolution image would be required to settle beyond doubt whether or not CC1 really exists. In the meantime we keep this object in our companion candidate list. HD 134180 may thus be a triple system and this may explain the slight disagreement between the NACO and the 2MASS astrometric data.

HD 145666. HD 145666 is surrounded by five companion candidates, three closer than $3.5^{\prime \prime}$ and two wider ones. These latter are listed in 2MASS and are unbound. We did not find preexisting data for the three closest objects, but the galactic coordinates of $\operatorname{HD} 145666\left(l=328.5^{\circ}, b=-4.0^{\circ}\right)$ suggest that they all belong to a population of background stars $\left(P_{\mathrm{ca}}(\mathrm{CC} 1)=3.1 \%\right.$, $\left.P_{\mathrm{ca}}(\mathrm{CC} 2)=7.1 \%, P_{\mathrm{ca}}(\mathrm{CC} 2+\mathrm{CC} 3)=13.0 \%\right)$.

HD 149606. We found five companion candidates in the vicinity of HD 149606. Not surprisingly $\left(l=342.2^{\circ}, b=+4.2^{\circ}\right)$, all of them are background stars.

HD 149612. HD 149612 has two companion candidates, one about 5.7" south-east, the other 7.89" north-west. The first object is clearly unbound, while the companionship status of the second is as yet unknown. The probability to have two unrelated objects with a magnitude $K \sim 12$ within $7.85^{\prime \prime}$ of HD 149612 is only of $1.8 \times 10^{-4}$. CC2 might thus be a true companion and a second-epoch observation of this system would be highly desirable. 

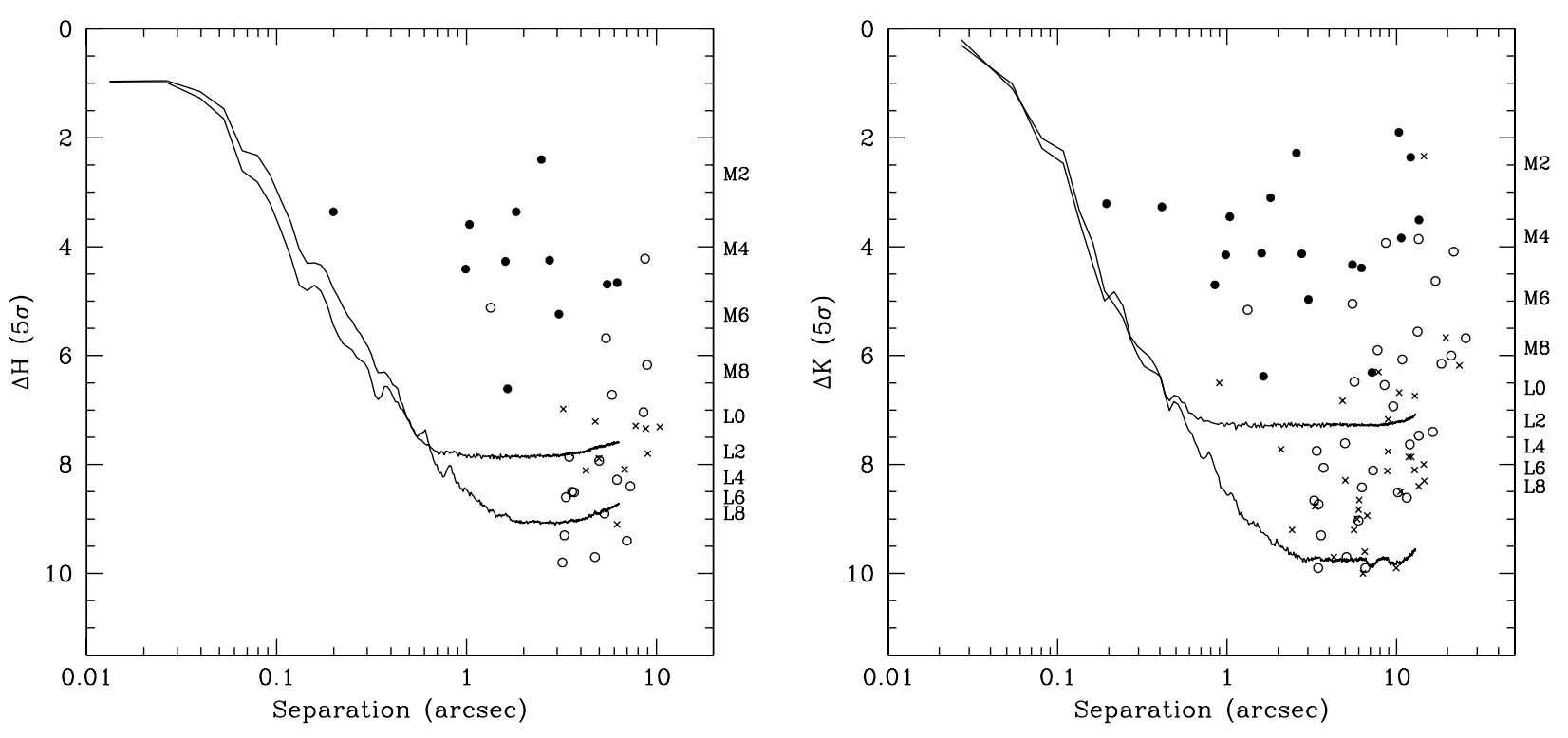

Fig. 5. Survey sensitivity and detected sources in the $H$ (left) and $K$ (right) bands. Dots represent bound and likely bound companions, open circles represent unbound and likely unbound companion candidates, while crosses denote companion candidates with an unknown companionship status. Labels on the right-hand side of each plot indicate the relationship between magnitude (NACO photometric system) and spectral type for companions to a typical K0 star. Solid lines are median sensitivity limits obtained with the two consecutive detectors of CONICA. The best detection curves in the external region dominated by readout noise are those obtained with the new Aladdin 3 detector.

HD 154682. Being close to the galactic plane and in the direction of the galactic center, HD 154682 is surrounded by 12 companion candidates. The closest $\left(0.99^{\prime \prime}\right)$ and brightest one is a true companion and will be denoted HD $154682 \mathrm{~B}$. Its colors are compatible with an M1.5-M4.5 spectral type and a mass of $0.27 \pm 0.02 M_{\odot}$. CC2 is an unrelated object, while CC5 is likely unbound. Combining our one-epoch data for CC11 and $\mathrm{CC} 12$ with their respective 2MASS measurements shows that $\mathrm{CC} 11$ is likely a background object, while $\mathrm{CC} 12$ is more likely unrelated $(0.7 \sigma)$ than bound $(1.7 \sigma)$. All the remaining companion candidates are without second epoch but they are probably background stars $\left(P_{\mathrm{ca}}(\mathrm{CC} 3)=37 \%, P_{\mathrm{ca}}(\mathrm{CC} 4)=20 \%\right.$, $P_{\mathrm{ca}}(\mathrm{CC} 6+\mathrm{CC} 3)=27 \%, \quad P_{\mathrm{ca}}(\mathrm{CC} 7+\mathrm{CC} 6+\mathrm{CC} 3)=21 \%, \quad P_{\mathrm{ca}}$ $\left.(\mathrm{CC} 10)=29 \%, P_{\mathrm{ca}}\left(>3 \mathrm{CCs}, \mathrm{K} \sim 15.5, \rho \leq 9.99^{\prime \prime}\right)=75 \%\right)$.

HD 171587. HD 171587 is located at low galactic latitude $\left(b=-1.6^{\circ}\right.$ ), only $21^{\circ}$ from the galactic center. Not surprisingly, we detected 12 nearby objects suggestive of a background-star population. The two closest objects (CC1 and $\mathrm{CC} 2$ ) are unbound (see Fig. 1 for CC2), while CC3 is likely unbound. The six widest candidates are listed in the 2MASS catalog and the combined astrometry show that they are all background stars. The remaining three candidates with only one observing epoch are also likely unbound.

HD 204313. A faint point source is present on our images 6.22 " south of HD 204313. We did not find additional data in the literature to check for common proper motion. HD 204313 is quite far from the galactic plane $\left(b=-43.7^{\circ}\right)$, but it is in the direction of the galactic center $\left(l=28.0^{\circ}\right)$. This implies a likelihood of chance alignment $P_{\text {ca }}=9.8 \%$.

HD 223913. HD 223913 has a known stellar companion (IDS 23484-6630B) about 12" south-east (WDS). Both components are listed in the 2MASS catalog and have uncontaminated photometry. The combined astrometric data show that this system is indeed bound, in agreement with the fact that the orbital parameters have not changed significantly over the last century (the WDS measurements are from the early 1900s). The near-infrared colors of HD 223913 B indicate a K9.5-M2.5 spectral type and a mass of $0.53 \pm 0.01 M_{\odot}$.

\subsection{Survey sensitivity}

We characterized the sensitivity of our data to companion detection by computing detection limits for point sources as a function of angular separation on the final image of each target (i.e. without PSF subtraction). Specifically, on each image we measured the standard deviation on concentric annuli (1 pixel wide) surrounding the target, masking companions and ghosts whenever present. A hypothetical point source located at a given radius was then said to be detectable when its maximum flux on a single pixel was larger than 5 times the standard deviation computed at this radius. This $5 \sigma$ detection threshold was checked a posteriori using the results obtained for the faintest companion candidates, and found to be indeed realistic. The image size used for the computation of detection limits was fixed to 968 pixels, corresponding to separations up to $6.3^{\prime \prime}$ for the S13 camera and up to $13^{\prime \prime}$ for the S27 camera. Several companions were detected at larger radii, but these larger separations were not systematically surveyed for all targets and in all directions.

A summary of the survey sensitivity is given in Fig. 5 for the two filters used for companion detection (NB_1.64 and NB_2.17). As noted in Sect. 3, the original detector of CONICA was replaced in the middle of our program, affecting the sensitivity limits (the observing strategy remained unchanged). The better quality of the new Aladdin3 detector is visible in Fig. 5, the sensitivity being improved in the outer regions dominated by readout noise. Generally speaking, our observations have a detection limit of $\Delta H \sim 5 \mathrm{mag}$ or $\Delta K \sim 5 \mathrm{mag}$ at $0.2^{\prime \prime}$, and $\Delta H \sim 8 \mathrm{mag}$ or $\Delta K \sim 7.5 \mathrm{mag}$ above $0.65^{\prime \prime}$. For G0 primaries, the contrasts reached at $0.2^{\prime \prime}$ allow the detection of companions down to M4-M5 dwarfs, while above $0.65^{\prime \prime}$ all M dwarf companions are detected. For K0 primaries, we typically detect companions down to M5-M6 at 0.2" and we reach the substellar 
domain above $0.65^{\prime \prime}$. Our survey thus probes a very large fraction of the mass domain occupied by potential stellar companions to solar-type planet-host stars and provides us with a very complete picture of the multiplicity status of these particular stars.

\section{Summary and concluding remarks}

Using the VLT/NACO adaptive optics facility we conducted a survey for stellar companions to 57 planet-host stars and to 73 control stars showing no obvious evidence for planetary companions from radial-velocity measurements. Our data reveal 95 companion candidates found in the vicinity of 33 of our targets. On the basis of multiepoch astrometry and colormagnitude diagrams, we identified 19 true companions, 2 likely bound objects, and 34 likely background stars. The remaining 40 companion candidates either lack second-epoch measurements or have inconclusive astrometric results. Although most of these candidates are likely background sources, a few may be true companions and we are carrying out second-epoch measurements to identify these few additional bound objects.

Among planet-host stars, we discovered two new companions to HD 65216, one to HD 177830, and we resolved the previously known companion to HD 196050 into a close pair of M dwarfs. The two companions to HD 65216 have been identified independently by Mugrauer et al. (2007) and both analyses show that they are very low mass stars at the bottom of the main sequence. HD 65216 therefore joins the small group of triple star systems hosting a planet. The same is true for HD 196050, which was previously thought to be a binary (Mugrauer et al. 2005a), but turned out to be a triple when observed at the NACO resolution. Like the HD 178911 system, HD 65216 and HD 196050 are made of a single planet-host star in orbit with a more distant binary, but contrary to HD 178911 they contain stars with very different masses ( $G$ primaries, $M / L$ secondaries and tertiaries). As the discovery of such systems requires high angular resolution and high-contrast data, it is not very surprising that we found two in our NACO survey whereas none were previously known (or recognized as such). The newly found companion to HD 177830 is an early M dwarf located at a projected separation of $97 \mathrm{AU}$.

Aside from these discoveries, our data also confirm the bound nature of the companions to the planet-bearing stars HD 142, HD 16141, and HD 46375, along with the unbound nature of a close $\left(\rho=1.3^{\prime \prime}\right)$ and relatively bright $(K=11.8)$ companion candidate to HD 162020 . We also detected potential companions near the planet-host stars HD 76700, HD 83443, HD 168746, and HD 330075. These companions are likely unrelated, but second-epoch measurements will be required to confirm this.

Among control stars, we discovered true companions to HD 7895, HD 24331, HD 31412, HD 43834, HD 78351, HD 104263, HD 129642, and HD 154682, and we confirm the bound status of the known companion candidates to HD 40397 , HD 70923, and HD 223913. Furthermore, we detected likely bound companions to HD 82241 and HD 134180. All these objects are either late-K stars or M dwarfs and have projected separations between 7 and 505 AU. HD 7895 and HD 31412 are moreover triple systems, with additional companions at about $800 \mathrm{AU}$.

The typical sensitivity of our survey enabled us to detect stellar companions down to the L-dwarf domain above $0.65^{\prime \prime}$, providing us with a very complete census of the stellar multiplicity among our 130 targets. This observational material forms an unprecedented data set to study the impact of stellar duplicity on planet occurrence and properties. Yet, as already mentioned, Doppler planet searches are biased in some ways against the closest binaries, and these selection effects must be taken into account to properly analyze the impact of stellar duplicity on planet occurrence. Although the introduction of a control subsample reduces to their lowest value the corrections related to these selection effects, some corrections will still be needed to ensure that both sub-samples are properly defined and can really be compared one to another. To be as free as possible from biases, we must also add to our sub-sample of planet-host stars the targets we did not include in our program because they were part of other (deeper) adaptive optics surveys. Since observers often tend to choose the most promising targets in terms of companion detection, these deliberate omissions might introduce some systematics on the statistical results if they are not compensated for. All these issues will be addressed in some detail in the second paper in this series, which will present a comprehensive statistical analysis aimed at investigating the impact of stellar duplicity on planet occurrence in binaries with projected separations between $\sim 20$ and $\sim 230$ AU.

Acknowledgements. We acknowledge support from the Swiss National Research Foundation (FNRS) and the Geneva University. This research was partly funded by the French INSU Programme National de Planétologie. We thank the ESO staff for support during the observations. We thank X. Delfosse and T. Forveille for useful discussions and for providing us with the list of their well-defined sample of $\mathbf{M}$ dwarfs. This publication has made use of data products from the Two Micron All Sky Survey, which is a joint project of the University of Massachusetts and the Infrared Processing and Analysis Center/California Institute of Technology, funded by the National Aeronautics and Space Administration and the National Science Foundation. This research has made use of the SIMBAD database and the VizieR catalog access tool operated at CDS, Strasbourg, France.

\section{References}

Abazajian, K., Adelman-McCarthy, J. K., Agüeros, M. A., et al. 2005, AJ, 129, 1755

Allen, C., Poveda, A., \& Herrera, M. A. 2000, A\&A, 356, 529

Artymowicz, P., \& Lubow, S. H. 1994, ApJ, 421, 651

Baraffe, I., Chabrier, G., Allard, F., \& Hauschildt, P. H. 1998, A\&A, 337, 403 Baranne, A., Mayor, M., \& Poncet, J. L. 1979, Vistas in Astronomy, 23, 279 Boss, A. P. 2006, ApJ, 641, 1148

Burrows, A., Sudarsky, D., \& Hubeny, I. 2006, ApJ, 640, 1063

Butler, R. P., Marcy, G. W., Williams, E., Hauser, H., \& Shirts, P. 1997, ApJ, 474, L115

Chabrier, G., Baraffe, I., Allard, F., \& Hauschildt, P. 2000, ApJ, 542, 464

Chauvin, G., Lagrange, A.-M., Udry, S., et al. 2006, A\&A, 456, 1165

Chen, B., Vergely, J. L., Valette, B., \& Carraro, G. 1998, A\&A, 336, 137

Chiu, K., Fan, X., Leggett, S. K., et al. 2006, AJ, 131, 2722

Cochran, W. D., Hatzes, A. P., Butler, R. P., \& Marcy, G. W. 1997, ApJ, 483, 457

Cox, A. N. 2000, Allen's astrophysical quantities, 4th edn. (New York: AIP Press, Springer), ed. A. N. Cox

Dahn, C. C., Harris, H. C., Vrba, F. J., et al. 2002, AJ, 124, 1170

Delfosse, X., Forveille, T., Ségransan, D., et al. 2000, A\&A, 364, 217

Devillard, N. 1997, The messenger, 87, 19

Duquennoy, A., \& Mayor, M. 1991, A\&A, 248, 485

Durisen, R. H., Boss, A. P., Mayer, L., et al. 2007, in Protostars and Planets V, ed. B. Reipurth, D. Jewitt, \& K. Keil, 607

Eggenberger, A., \& Udry, S. 2007, in Planets in Binary Star Systems, ed. N. Haghighipour (Springer Publishing Company), in press, [arXiv: astro-ph:0705.3173]

Eggenberger, A., Udry, S., \& Mayor, M. 2003, in Scientific Frontiers in Research on Extrasolar Planets, 43, ASP Conf. Ser., 294

Eggenberger, A., Halbwachs, J.-L., Udry, S., \& Mayor, M. 2004a, Rev. Mex. Astron. Astrof. Conf. Ser., ed. C. Allen, \& C. Scarfe, 28

Eggenberger, A., Udry, S., \& Mayor, M. 2004b, A\&A, 417, 353

Eggenberger, A., Udry, S., Mayor, M., et al. 2004c, in Extrasolar Planets: Today and Tomorrow, ed. J. Beaulieu, A. Lecavelier Des Etangs, \& C. Terquem, ASP Conf. Ser. 321, 93 
Eggenberger, P., Carrier, F., \& Bouchy, F. 2005, New Astron., 10, 195

Eggenberger, A., Udry, S., Mayor, M., et al. 2007, in Multiple Stars Across the H-R Diagram, ESO Astrophysic Symposia, ed. S. Hubrig, M. Petr-Gotzens, \& A. Tokovinin, in Press, available at www. eso.org/gen-fac/meetings/ms2005/eggenberger.pdf

Geballe, T. R., Knapp, G. R., Leggett, S. K., et al. 2002, ApJ, 564, 466

Halbwachs, J. L., Mayor, M., Udry, S., \& Arenou, F. 2003, A\&A, 397, 159

Hartkopf, W. I., \& Mason, B. D. 2004, in Rev. Mex. Astron. Astrof. Conf. Ser., ed. C. Allen, \& C. Scarfe, 83

Hartkopf, W. I., Mason, B. D., \& Worley, C. E. 2001, AJ, 122, 3472

Knapp, G. R., Leggett, S. K., Fan, X., et al. 2004, AJ, 127, 3553

Konacki, M. 2005, ApJ, 626, 431

Leggett, S. K., Allard, F., Dahn, C., et al. 2000, ApJ, 535, 965

Leggett, S. K., Allard, F., Geballe, T. R., Hauschildt, P. H., \& Schweitzer, A. 2001, ApJ, 548, 908

Leggett, S. K., Golimowski, D. A., Fan, X., et al. 2002, ApJ, 564, 452

Lenzen, R., Hartung, M., Brandner, W., et al. 2003, in Instrument Design and Performance for Optical/Infrared Ground-based Telescopes, ed. I. M. Moorwood, F. M. Alan, M. Iye, \& A. F. M. Moorwood, Proc. SPIE, 4841, 944

Lissauer, J. J., \& Stevenson, D. J. 2007, in Protostars and Planets V, ed. B. Reipurth, D. Jewitt, \& K. Keil, 591

Luhman, K. L., \& Jayawardhana, R. 2002, ApJ, 566, 1132

Mayer, L., Wadsley, J., Quinn, T., \& Stadel, J. 2005, MNRAS, 363, 641

McCaughrean, M. J., \& Stauffer, J. R. 1994, AJ, 108, 1382

Meynet, G., \& Maeder, A. 2000, A\&A, 361, 101

Mugrauer, M., Neuhäuser, R., Mazeh, T., Alves, J., \& Guenther, E. 2004a, A\&A, 425,249

Mugrauer, M., Neuhäuser, R., Mazeh, T., Guenther, E., \& Fernández, M. 2004b, Astron. Nachr., 325, 718

Mugrauer, M., Neuhaeuser, R., Guenther, E., \& Mazeh, T. 2005a, Astron. Nachr., 326, 629
Mugrauer, M., Neuhäuser, R., Seifahrt, A., Mazeh, T., \& Guenther, E. 2005b, A\&A, 440, 1051

Mugrauer, M., Neuhäuser, R., Mazeh, T., et al. 2006, Astron. Nachr., 327, 321

Mugrauer, M., Seifahrt, A., \& Neuhäuser, R. 2007, MNRAS, submitted Nelson, A. F. 2000, ApJ, 537, L65

Nordström, B., Mayor, M., Andersen, J., et al. 2004, A\&A, 418, 989

Patience, J., White, R. J., Ghez, A. M., et al. 2002, ApJ, 581, 654

Perrier, C., Sivan, J.-P., Naef, D., et al. 2003, A\&A, 410, 1039

Pichardo, B., Sparke, L. S., \& Aguilar, L. A. 2005, MNRAS, 359, 521

Pickles, A. J. 1998, PASP, 110, 863

Poveda, A., Herrera, M. A., Allen, C., Cordero, G., \& Lavalley, C. 1994, Rev. Mex. Astron. Astrofis., 28, 43

Queloz, D., Mayor, M., Weber, L., et al. 2000, A\&A, 354, 99

Raghavan, D., Henry, T. J., Mason, B. D., et al. 2006, ApJ, 646, 523

Robin, A. C., Reylé, C., Derrière, S., \& Picaud, S. 2003, A\&A, 409, 523

Rousset, G., Lacombe, F., Puget, P., et al. 2003, in Adaptive Optical System

Technologies II, ed. P. L. Wizinowich, \& D. Bonaccini, Proc. SPIE, ed. P. L.

Wizinowich, \& D. Bonaccini, 4839, 140

Santos, N. C., Israelian, G., \& Mayor, M. 2004, A\&A, 415, 1153

Santos, N. C., Israelian, G., Mayor, M., et al. 2005, A\&A, 437, 1127

Skrutskie, M. F., Cutri, R. M., Stiening, R., et al. 2006, AJ, 131, 1163

Tokovinin, A., Thomas, S., Sterzik, M., \& Udry, S. 2006, A\&A, 450, 681

Udry, S., Eggenberger, A., Beuzit, J.-L., et al. 2004, in Rev. Mex. Astron. Astrofis. Conf. Ser., ed. C. Allen, \& C. Scarfe, 215

Udry, S., Mayor, M., Naef, D., et al. 2000, A\&A, 356, 590

van Dessel, E., \& Sinachopoulos, D. 1993, A\&AS, 100, 517

Veran, J.-P., \& Rigaut, F. J. 1998, in Adaptive Optical System Technologies, ed. D. Bonaccini, \& R. K. Tyson, Proc. SPIE, 3353, 426

Vrba, F. J., Henden, A. A., Luginbuhl, C. B., et al. 2004, AJ, 127, 2948

Zucker, S., \& Mazeh, T. 2002, ApJ, 568, L113

Zucker, S., Mazeh, T., Santos, N. C., Udry, S., \& Mayor, M. 2003, A\&A, 404, 775 
A. Eggenberger et al.: The impact of stellar duplicity on planet occurrence and properties. I., Online Material $p 1$

\section{Online Material}


A. Eggenberger et al.: The impact of stellar duplicity on planet occurrence and properties. I., Online Material p 2

Table 1. Target list and target properties for the planet-host star sub-sample. Apparent magnitudes, proper motions and spectral types are from the Hipparcos catalog. Distances were computed from the Hipparcos parallaxes.

\begin{tabular}{|c|c|c|c|c|}
\hline Target & $\overline{\bar{V}}$ & $\begin{array}{c}\text { Distance } \\
(\mathrm{pc})\end{array}$ & $\begin{array}{c}\text { Proper motion } \\
\left(\text { mas yr }^{-1}\right)\end{array}$ & $\overline{\overline{\mathrm{SP}}}$ \\
\hline HD 142 & 5.70 & $25.64_{-0.41}^{+0.43}$ & $576.59 \pm 0.52$ & G1IV... \\
\hline HD 2039 & 9.00 & $89.85_{-8.28}^{+10.15}$ & $79.99 \pm 0.93$ & G2/G3IV/V \\
\hline HD 3651 & 5.88 & $11.11_{-0.09}^{+0.09}$ & $591.72 \pm 0.96$ & $\mathrm{~K} 0 \mathrm{~V}$ \\
\hline HD 4203 & 8.70 & $77.82_{-7.00}^{+8.53}$ & $176.24 \pm 1.34$ & G5 \\
\hline HD 4208 & 7.78 & $32.70_{-1.12}^{+1.20}$ & $347.55 \pm 1.16$ & G5V \\
\hline HD 4308 & 6.55 & $21.85_{-0.26}^{+0.27}$ & $758.99 \pm 0.61$ & G3V \\
\hline HD 6434 & 7.72 & $40.32_{-1.40}^{+1.50}$ & $554.10 \pm 0.77$ & G3IV \\
\hline HD 16141 & 6.83 & $35.91_{-1.71}^{+1.89}$ & $464.36 \pm 1.33$ & G5IV \\
\hline HD 23079 & 7.12 & $34.60_{-0.66}^{+0.68}$ & $214.33 \pm 0.78$ & F8/G0V \\
\hline HD 27894 & 9.36 & $42.37_{-1.57}^{+1.70}$ & $327.68 \pm 1.41$ & $\mathrm{~K} 2 \mathrm{~V}$ \\
\hline HD 28185 & 7.80 & $39.56_{-1.62}^{+1.77}$ & $100.85 \pm 1.30$ & G5 \\
\hline HD 30177 & 8.41 & $54.70_{-2.21}^{+2.41}$ & $67.20 \pm 1.03$ & G8V \\
\hline HD 33636 & 7.00 & $28.69_{-1.05}^{+1.14}$ & $227.06 \pm 1.36$ & G0 \\
\hline HD 38529 & 5.95 & $42.43_{-1.59}^{+1.72}$ & $162.82 \pm 0.97$ & G4V \\
\hline HD 39091 & 5.65 & $18.21_{-0.15}^{+0.15}$ & $1095.54 \pm 0.64$ & G3IV \\
\hline HD 46375 & 7.91 & $33.41_{-1.15}^{+1.24}$ & $149.73 \pm 1.17$ & K1IV \\
\hline HD 50554 & 6.84 & $31.03_{-0.94}^{+1.00}$ & $103.32 \pm 1.09$ & F8 \\
\hline HD 65216 & 7.97 & $35.59_{-0.85}^{+0.90}$ & $190.26 \pm 1.15$ & G5V \\
\hline HD 70642 & 7.17 & $28.76_{-0.49}^{+0.51}$ & $302.87 \pm 0.69$ & G8:III.. \\
\hline HD 73256 & 8.08 & $36.52_{-1.00}^{+1.06}$ & $192.16 \pm 0.58$ & G8/K0V \\
\hline HD 73526 & 8.99 & $94.61_{-8.25}^{+10.00}$ & $172.58 \pm 1.10$ & G6V \\
\hline HD 74156 & 7.61 & $64.56_{-4.28}^{+4.93}$ & $202.02 \pm 0.95$ & G0 \\
\hline HD 75289 & 6.35 & $28.94_{-0.46}^{+0.48}$ & $228.60 \pm 0.51$ & G0Ia0: \\
\hline HD 76700 & 8.16 & $59.70_{-2.26}^{+2.45}$ & $307.92 \pm 0.97$ & G8V \\
\hline HD 82943 & 6.54 & $27.46_{-0.62}^{+0.65}$ & $174.06 \pm 0.54$ & G0 \\
\hline HD 83443 & 8.23 & $43.54_{-1.64}^{+1.78}$ & $122.81 \pm 0.80$ & K0V \\
\hline HD 92788 & 7.31 & $32.32_{-1.00}^{+1.07}$ & $223.10 \pm 0.77$ & G5 \\
\hline HD 102117 & 7.47 & $42.00_{-1.41}^{+1.52}$ & $94.11 \pm 0.97$ & G6V \\
\hline HD 106252 & 7.41 & $37.44_{-1.23}^{+1.32}$ & $280.41 \pm 0.57$ & G0 \\
\hline HD 108147 & 6.99 & $38.57_{-1.00}^{+1.05}$ & $191.50 \pm 0.78$ & F8/G0V \\
\hline HD 108874 & 8.76 & $68.54_{-5.37}^{+6.37}$ & $157.08 \pm 1.41$ & G5 \\
\hline HD 111232 & 7.59 & $28.88_{-0.65}^{+0.68}$ & $116.19 \pm 0.78$ & G5V \\
\hline HD 114386 & 8.73 & $28.04_{-1.00}^{+1.08}$ & $353.26 \pm 1.68$ & $\mathrm{~K} 3 \mathrm{~V}$ \\
\hline HD 114729 & 6.68 & $35.00_{-1.15}^{+1.23}$ & $368.81 \pm 1.11$ & G0V \\
\hline HD 114783 & 7.56 & $20.43_{-0.43}^{+0.45}$ & $138.46 \pm 1.13$ & K0 \\
\hline HD 121504 & 7.54 & $44.37_{-1.72}^{+1.87}$ & $264.26 \pm 0.93$ & G2V \\
\hline HD 128311 & 7.48 & $16.57_{-0.27}^{+0.28}$ & $323.34 \pm 1.10$ & K0 \\
\hline HD 130322 & 8.04 & $29.76_{-1.28}^{+1.40}$ & $191.35 \pm 1.92$ & K0III \\
\hline HD 134987 & 6.47 & $25.65_{-0.63}^{+0.66}$ & $406.01 \pm 1.19$ & G5V \\
\hline HD 136118 & 6.93 & $52.27_{-2.22}^{+2.43}$ & $126.25 \pm 0.99$ & F8 \\
\hline HD 141937 & 7.25 & $33.46_{-1.17}^{+1.25}$ & $100.04 \pm 1.35$ & G2/G3V \\
\hline HD 142415 & 7.33 & $34.57_{-0.98}^{+1.03}$ & $153.17 \pm 1.11$ & G1V \\
\hline
\end{tabular}


A. Eggenberger et al.: The impact of stellar duplicity on planet occurrence and properties. I., Online Material $p 3$

Table 1. continued.

\begin{tabular}{lcccc}
\hline \hline Target & $V$ & $\begin{array}{c}\text { Distance } \\
(\mathrm{pc})\end{array}$ & $\begin{array}{c}\text { Proper motion } \\
\left(\mathrm{mas} \mathrm{yr}^{-1}\right)\end{array}$ & SP \\
\hline HD 147513 & 5.37 & $12.87_{-0.14}^{+0.14}$ & $72.72 \pm 0.86$ & G3/G5V \\
HD 162020 & 9.10 & $31.26_{-1.38}^{+1.52}$ & $32.79 \pm 2.47$ & K2V: \\
HD 168746 & 7.95 & $43.12_{-1.71}^{+1.86}$ & $72.68 \pm 0.90$ & G5 \\
HD 169830 & 5.90 & $36.32_{-1.16}^{+1.24}$ & $15.18 \pm 0.78$ & F8V \\
HD 177830 & 7.18 & $59.03_{-2.53}^{+2.77}$ & $65.89 \pm 0.81$ & K0 \\
HD 179949 & 6.25 & $27.05_{-0.57}^{+0.60}$ & $153.42 \pm 1.05$ & F8V \\
HD 196050 & 7.50 & $46.93_{-1.92}^{+2.09}$ & $201.49 \pm 0.76$ & G3V \\
HD 202206 & 8.08 & $46.34_{-2.33}^{+2.58}$ & $125.72 \pm 0.88$ & G6V \\
HD 210277 & 6.54 & $21.29_{-0.35}^{+0.36}$ & $457.86 \pm 0.65$ & G0 \\
HD 213240 & 6.81 & $40.75_{-1.30}^{+1.39}$ & $236.49 \pm 0.76$ & G4IV \\
HD 216435 & 6.03 & $33.29_{-0.79}^{+0.83}$ & $231.51 \pm 0.65$ & G3IV \\
HD 216437 & 6.04 & $26.52_{-0.40}^{+0.41}$ & $84.99 \pm 0.54$ & G4IV-V \\
HD 216770 & 8.11 & $37.89_{-1.46}^{+1.59}$ & $289.83 \pm 1.44$ & K0V \\
HD 222582 & 7.68 & $41.95_{-1.87}^{+2.05}$ & $182.99 \pm 1.47$ & G5 \\
HD 330075 & 9.36 & $50.20_{-3.49}^{+4.06}$ & $253.69 \pm 1.69$ & G5 \\
\hline
\end{tabular}


A. Eggenberger et al.: The impact of stellar duplicity on planet occurrence and properties. I., Online Material p 4

Table 2. Target list and target properties for the control sub-sample. Apparent magnitudes, proper motions and spectral types are from the Hipparcos catalog. Distances were computed from the Hipparcos parallaxes.

\begin{tabular}{|c|c|c|c|c|}
\hline Target & $\overline{\bar{V}}$ & $\begin{array}{c}\text { Distance } \\
(\mathrm{pc})\end{array}$ & $\begin{array}{c}\text { Proper motion } \\
\left(\text { mas yr }^{-1}\right)\end{array}$ & $\overline{\overline{\mathrm{SP}}}$ \\
\hline HD 4391 & 5.80 & $14.94_{-0.16}^{+0.16}$ & $199.95 \pm 0.69$ & G5IV \\
\hline HD 6107 & 6.91 & $44.98_{-1.26}^{+1.33}$ & $122.37 \pm 0.80$ & $\mathrm{G} 1 / \mathrm{G} 2 \mathrm{~V}$ \\
\hline HD 6715 & 7.61 & $32.43_{-088}^{+0.93}$ & $403.76 \pm 0.92$ & G5 \\
\hline HD 7895 & 8.00 & $27.65_{-0.74}^{+0.79}$ & $498.52 \pm 1.29$ & $\mathrm{~K} 1 \mathrm{~V}$ \\
\hline HD 8638 & 8.29 & $40.40_{-1.90}^{+2.09}$ & $423.26 \pm 1.03$ & G6Vw.. \\
\hline HD 8859 & 8.41 & $40.80_{-1.58}^{+1.72}$ & $191.40 \pm 0.79$ & G5V \\
\hline HD 11112 & 7.13 & $45.13_{-1.52}^{+1.62}$ & $441.22 \pm 0.72$ & G4V \\
\hline HD 12042 & 6.10 & $26.34_{-0.42}^{+0.43}$ & $428.79 \pm 0.74$ & F8V \\
\hline HD 18194 & 8.33 & $34.32_{-1.27}^{+1.37}$ & $203.54 \pm 1.42$ & G5 \\
\hline HD 19034 & 8.08 & $35.30_{-1.43}^{+1.56}$ & $425.69 \pm 1.58$ & G5 \\
\hline HD 20201 & 7.27 & $37.54_{-0.91}^{+0.95}$ & $92.23 \pm 0.85$ & G0V \\
\hline HD 22610 & 9.43 & $40.72_{-1.49}^{+1.60}$ & $96.45 \pm 1.42$ & $\mathrm{~K} 2 \mathrm{~V}$ \\
\hline HD 24045 & 8.52 & $37.95_{-1.65}^{+1.81}$ & $81.42 \pm 1.30$ & G8V \\
\hline HD 24331 & 8.61 & $26.70_{-0.59}^{+0.62}$ & $653.38 \pm 1.17$ & $\mathrm{~K} 2 \mathrm{~V}$ \\
\hline HD 25105 & 8.53 & $35.89_{-1.09}^{+1.16}$ & $106.80 \pm 0.82$ & K0V \\
\hline HD 30278 & 7.61 & $27.22_{-0.44}^{+0.45}$ & $204.81 \pm 0.78$ & G8V \\
\hline HD 30670 & 9.28 & $37.95_{-1.32}^{+1.42}$ & $59.22 \pm 1.22$ & K3V \\
\hline HD 31412 & 7.02 & $36.00_{-1.13}^{+1.21}$ & $227.46 \pm 1.11$ & F8 \\
\hline HD 38858 & 5.97 & $15.56_{-0.28}^{+0.29}$ & $238.93 \pm 1.02$ & G4V \\
\hline HD 40397 & 6.99 & $23.20_{-0.49}^{+0.51}$ & 215.4 & G0 \\
\hline HD 43834 & 5.08 & $10.15_{-0.05}^{+0.05}$ & $245.22 \pm 0.60$ & G5V \\
\hline HD 45067 & 5.88 & $33.09_{-0.98}^{+1.04}$ & $322.99 \pm 1.07$ & F8V \\
\hline HD 54359 & 8.85 & $28.70_{-1.109}^{+1.17}$ & $201.17 \pm 1.40$ & K0 \\
\hline HD 55720 & 7.50 & $27.69_{-0.45}^{+0.46}$ & $818.02 \pm 0.65$ & G6V \\
\hline HD 56560 & 7.33 & $46.25_{-1.13}^{+1.18}$ & $283.88 \pm 0.81$ & G6IV/V \\
\hline HD 59468 & 6.72 & $22.51_{-0.27}^{+0.27}$ & $285.31 \pm 0.55$ & G5IV-V \\
\hline HD 67556 & 7.30 & $47.04_{-1.44}^{+1.53}$ & $107.01 \pm 0.68$ & F8V \\
\hline HD 70923 & 7.07 & $43.65_{-1.74}^{+1.89}$ & $206.97 \pm 1.16$ & G0 \\
\hline HD 71835 & 8.39 & $39.94_{-1.31}^{+1.40}$ & $99.30 \pm 0.91$ & G8/K0V \\
\hline HD 73524 & 6.53 & $27.47_{-0.42}^{+0.44}$ & $306.59 \pm 0.51$ & G1V \\
\hline HD 74385 & 8.11 & $22.36_{-0.39}^{+0.42}$ & $283.09 \pm 0.95$ & $\mathrm{~K} 2 \mathrm{~V}$ \\
\hline HD 78351 & 8.42 & $38.71_{-1.32}^{+1.41}$ & $61.86 \pm 0.83$ & G8V \\
\hline HD 78538 & 8.15 & $43.03_{-1.96}^{+2.16}$ & $89.33 \pm 1.46$ & G5 \\
\hline HD 78558 & 7.29 & $36.67_{-1.18}^{+1.27}$ & $570.54 \pm 1.00$ & G3V \\
\hline HD 82241 & 6.98 & $39.67_{-0.98}^{+1.03}$ & $96.86 \pm 0.66$ & F8V \\
\hline HD 85390 & 8.54 & $33.96_{-0.94}^{+1.00}$ & $64.67 \pm 0.94$ & K1V \\
\hline HD 98281 & 7.29 & $21.99_{-0.47}^{+0.49}$ & $809.04 \pm 1.24$ & G8V \\
\hline HD 101581 & 7.77 & $12.51_{-0.14}^{+0.15}$ & $701.36 \pm 0.77$ & $\mathrm{~K} 5 \mathrm{~V}$ \\
\hline HD 104263 & 8.23 & $42.81_{-1.91}^{+2.10}$ & $182.26 \pm 1.18$ & G5 \\
\hline HD 106116 & 7.43 & $33.90_{-1.01}^{+1.08}$ & $738.42 \pm 0.88$ & G4V \\
\hline HD 107885 & 7.75 & $49.88_{-2.10}^{+2.29}$ & $59.74 \pm 0.88$ & F8Vw... \\
\hline HD 108523 & 8.31 & $46.15_{-1.90}^{+2.07}$ & $303.61 \pm 1.05$ & G6V \\
\hline HD 114747 & 8.03 & $26.70_{-0.76}^{+0.81}$ & $255.60 \pm 1.35$ & $\mathrm{~K} 1 \mathrm{IV} / \mathrm{V}$ \\
\hline HD 115585 & 7.43 & $43.38_{-1.28}^{+1.36}$ & $393.02 \pm 0.69$ & G6IV-V \\
\hline
\end{tabular}


A. Eggenberger et al.: The impact of stellar duplicity on planet occurrence and properties. I., Online Material p 5

Table 2. continued.

\begin{tabular}{|c|c|c|c|c|}
\hline Target & $\overline{\bar{V}}$ & $\begin{array}{c}\text { Distance } \\
(\mathrm{pc})\end{array}$ & $\begin{array}{c}\text { Proper motion } \\
\left(\text { mas yr }^{-1}\right)\end{array}$ & $\overline{\overline{\mathrm{SP}}}$ \\
\hline HD 117105 & 7.20 & $35.69_{-1.22}^{+1.31}$ & $310.59 \pm 1.33$ & G1V \\
\hline HD 123265 & 8.35 & $38.26_{-1.65}^{+1.81}$ & $420.01 \pm 1.30$ & K0 \\
\hline HD 125455 & 7.58 & $20.78_{-0.47}^{+0.49}$ & $643.43 \pm 1.28$ & $\mathrm{~K} 1 \mathrm{~V}$ \\
\hline HD 125881 & 7.26 & $36.44_{-1.09}^{+1.16}$ & $291.87 \pm 0.86$ & G2V \\
\hline HD 129642 & 8.40 & $28.59_{-0.92}^{+0.98}$ & $769.08 \pm 1.13$ & $\mathrm{~K} 3 \mathrm{~V}$ \\
\hline HD 134180 & 9.41 & $37.15_{-1.92}^{+2.15}$ & $204.03 \pm 1.75$ & $\mathrm{~K} 3 \mathrm{~V}$ \\
\hline HD 136257 & 7.53 & $44.88_{-2.00}^{+2.20}$ & $225.18 \pm 1.19$ & F9V \\
\hline HD 143114 & 7.34 & $36.09_{-1.15}^{+1.23}$ & $89.16 \pm 1.12$ & G3V \\
\hline HD 145518 & 7.41 & $33.58_{-1.11}^{+1.19}$ & $52.22 \pm 1.29$ & $\mathrm{G} 1 / \mathrm{G} 2 \mathrm{~V}$ \\
\hline HD 145666 & 7.80 & $41.44_{-1.62}^{+1.75}$ & $309.09 \pm 1.36$ & G2IV \\
\hline HD 146775 & 7.68 & $41.19_{-1.66}^{+1.81}$ & $260.07 \pm 0.95$ & G5V \\
\hline HD 149606 & 8.95 & $32.37_{-1.37}^{+1.50}$ & $537.47 \pm 1.71$ & $\mathrm{~K} 2 \mathrm{~V}$ \\
\hline HD 149612 & 7.01 & $21.68_{-0.42}^{+0.44}$ & $364.73 \pm 0.91$ & G3V \\
\hline HD 153713 & 9.45 & $42.44_{-2.89}^{+3.34}$ & $94.10 \pm 1.78$ & $\mathrm{~K} 1 \mathrm{~V}$ \\
\hline HD 154682 & 7.68 & $45.79_{-2.16}^{+2.38}$ & $95.91 \pm 1.26$ & G5V \\
\hline HD 162396 & 6.19 & $32.73_{-0.94}^{+0.99}$ & $237.51 \pm 1.04$ & F8V \\
\hline HD 170493 & 8.04 & $18.76_{-0.36}^{+0.38}$ & $259.19 \pm 1.18$ & $\mathrm{~K} 3 \mathrm{~V}$ \\
\hline HD 171587 & 8.52 & $41.53_{-2.10}^{+2.33}$ & $231.55 \pm 1.32$ & G5 \\
\hline HD 184509 & 6.74 & $31.65_{-1.06}^{+1.14}$ & $195.36 \pm 0.90$ & G1V \\
\hline HD 189567 & 6.07 & $17.71_{-0.23}^{+0.24}$ & $1080.95 \pm 0.66$ & $\mathrm{G} 2 \mathrm{~V}$ \\
\hline HD 192417 & 8.20 & $47.62_{-2.19}^{+2.41}$ & $51.56 \pm 1.06$ & G3V \\
\hline HD 204313 & 7.99 & $47.30_{-2.26}^{+2.50}$ & $274.38 \pm 0.69$ & G5V \\
\hline HD 212168 & 6.12 & $23.05_{-0.50}^{+0.52}$ & $59.86 \pm 1.12$ & G3IV \\
\hline HD 213575 & 6.94 & $35.82_{-1.06}^{+1.12}$ & $292.99 \pm 0.91$ & G0 \\
\hline HD 214759 & 7.41 & $26.70_{-0.64}^{+0.67}$ & $344.05 \pm 0.78$ & G8/K0V \\
\hline HD 215456 & 6.63 & $38.64_{-1.14}^{+1.22}$ & $212.86 \pm 0.67$ & F9V \\
\hline HD 215657 & 7.22 & $32.89_{-0.96}^{+1.02}$ & $35.71 \pm 1.05$ & G3IV-V \\
\hline HD 220476 & 7.62 & $30.21_{-0.81}^{+0.85}$ & $144.11 \pm 0.97$ & G5 \\
\hline HD 223913 & 6.64 & $25.87_{-0.41}^{+0.42}$ & $95.23 \pm 0.72$ & G0V \\
\hline
\end{tabular}


A. Eggenberger et al.: The impact of stellar duplicity on planet occurrence and properties. I., Online Material p 6
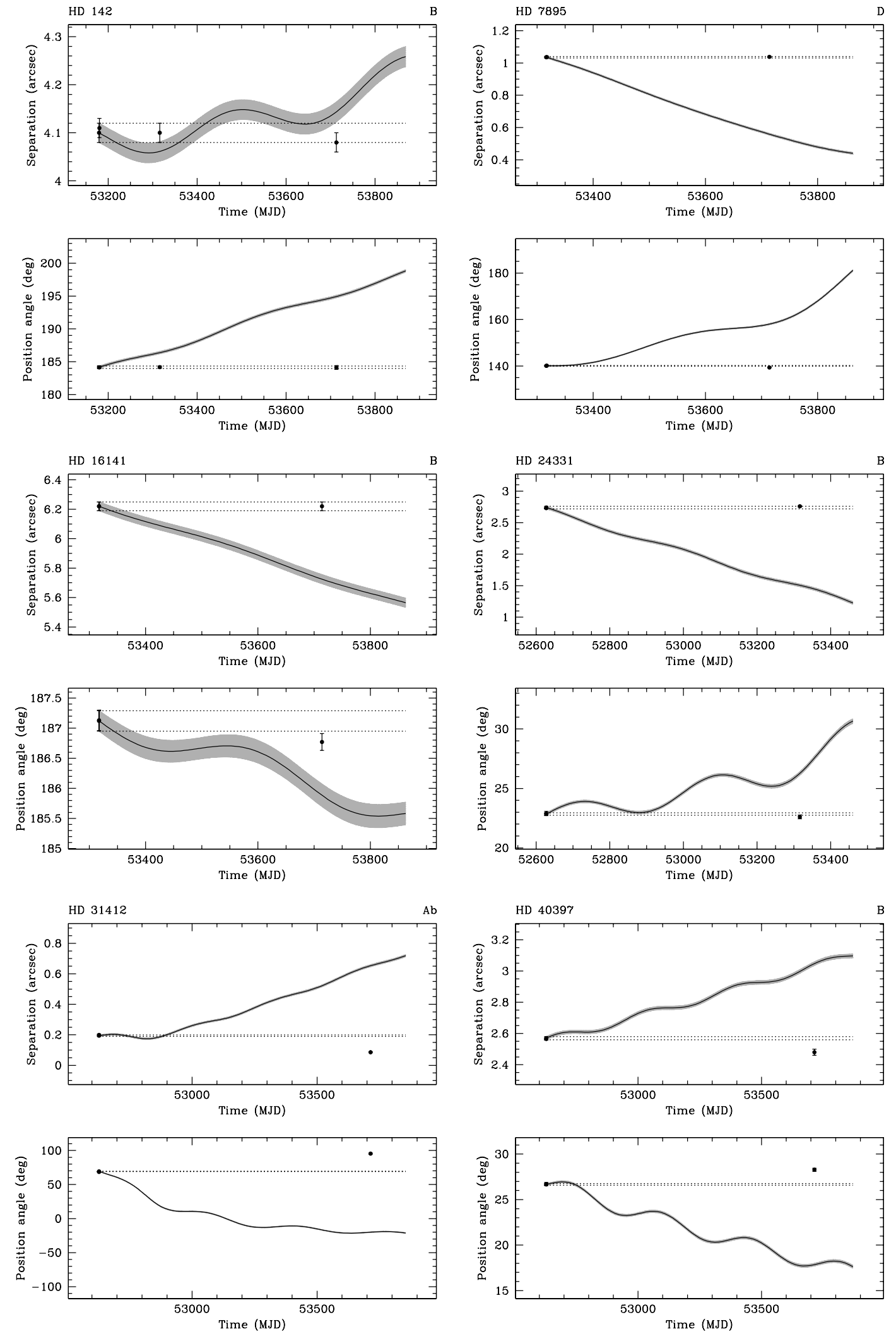

Fig. 3. Multiepoch astrometry for the true and likely bound companions. For each companion candidate, the upper panel shows the evolution of angular separation versus time, and the lower panel the evolution of position angle versus time. Solid lines take into account the primary proper and parallactic motions, and thus depict the evolution expected for background objects with negligible proper motion. The grey zones represent the associated uncertainties, which comprise uncertainties on the parallax and on the proper motion of the primary star, along with the uncertainty on the first-epoch measurement. Our own data points are represented by dots, while additional measurements from the literature (see the notes for references) are represented by crosses. 
A. Eggenberger et al.: The impact of stellar duplicity on planet occurrence and properties. I., Online Material p 7
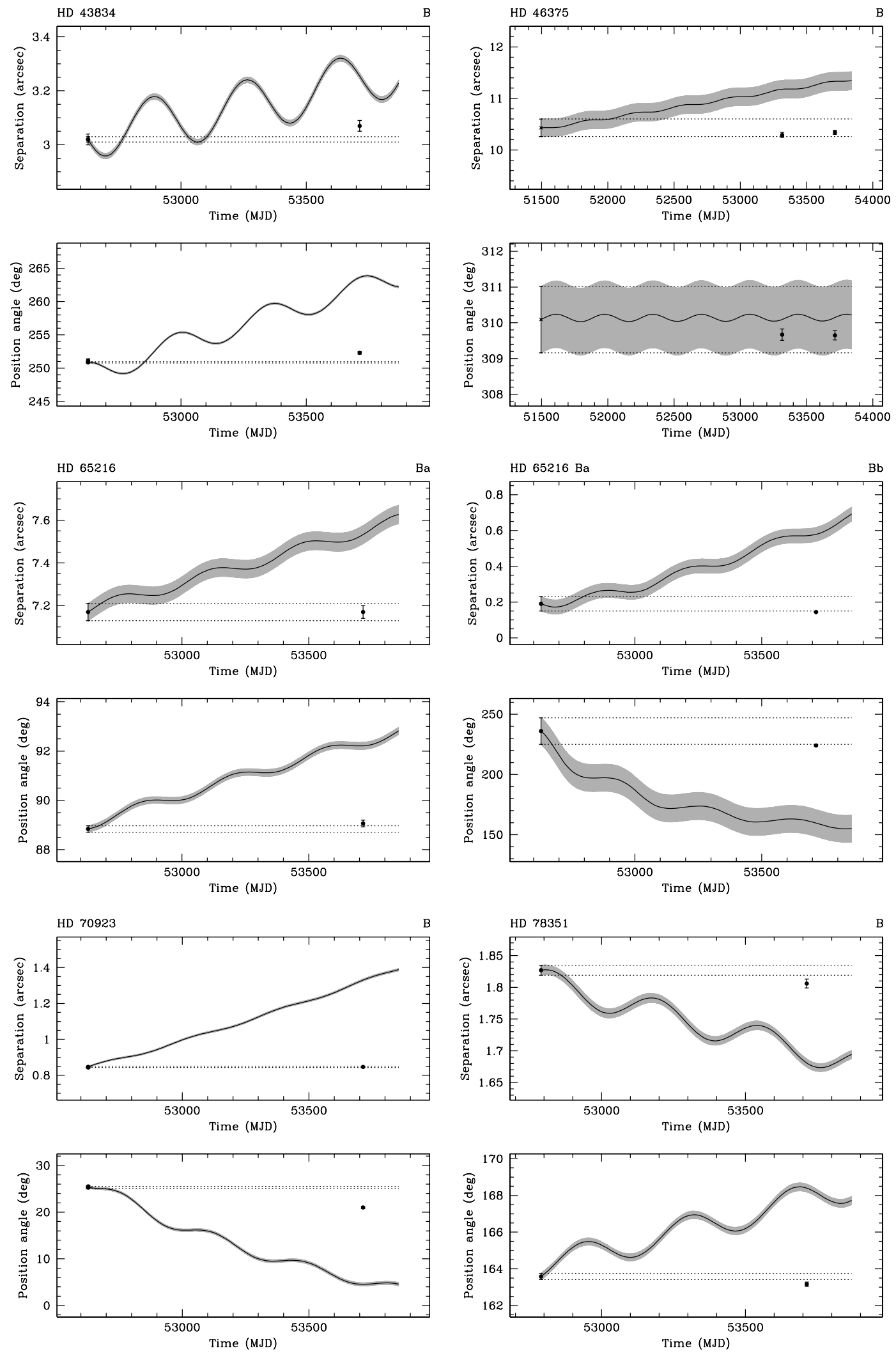

Fig. 3. continued. 
A. Eggenberger et al.: The impact of stellar duplicity on planet occurrence and properties. I., Online Material $p 8$
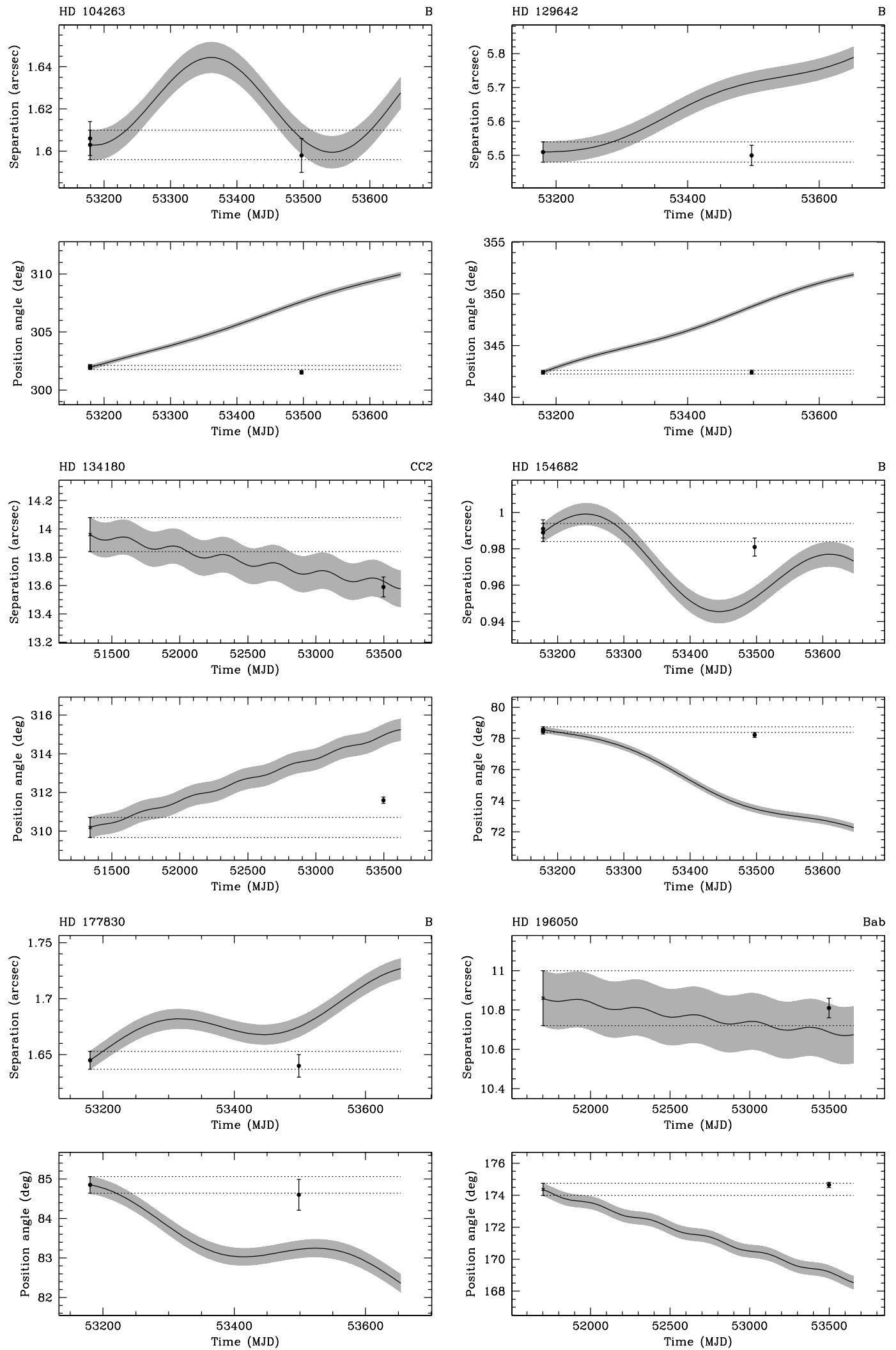

Fig. 3. continued. 
A. Eggenberger et al.: The impact of stellar duplicity on planet occurrence and properties. I., Online Material p 9
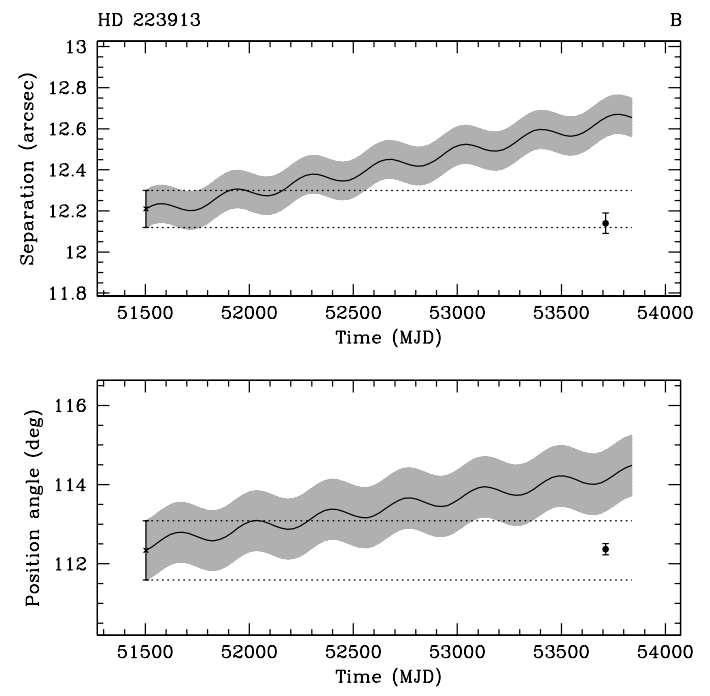

Fig. 3. continued. 
A. Eggenberger et al.: The impact of stellar duplicity on planet occurrence and properties. I., Online Material p 10

HD 142

NB_2.17 HD 7895

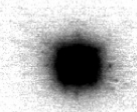

2"

HD 24331
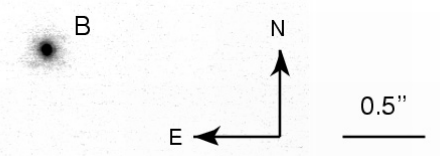

NB_1.64

HD 31412

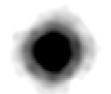

NB_1.64 HD 16141

NB_2.17

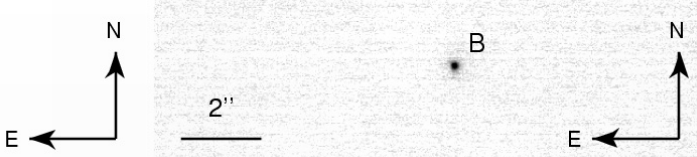

NB 2.17 HD 40397

NB 1.64
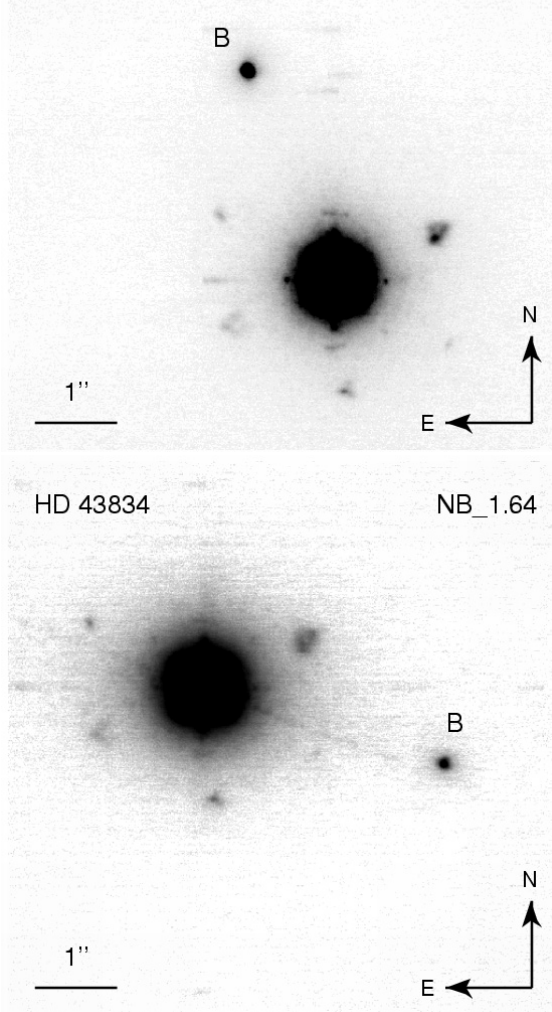

NB_2.17

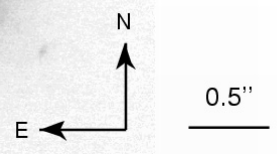

HD 46375

$A b$

$\mathrm{Aa}$
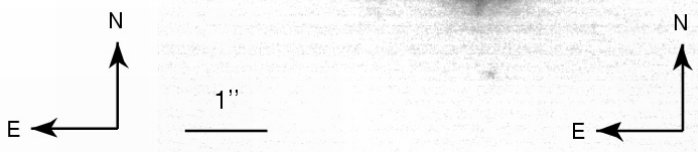

NB_2.17

HD 65216

NB_2.17

B

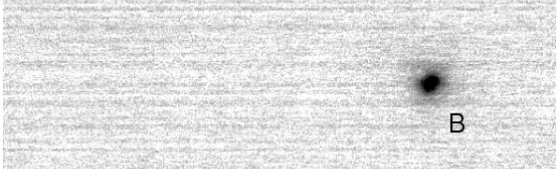

$\mathrm{Ba}$ $\mathrm{Bb}$
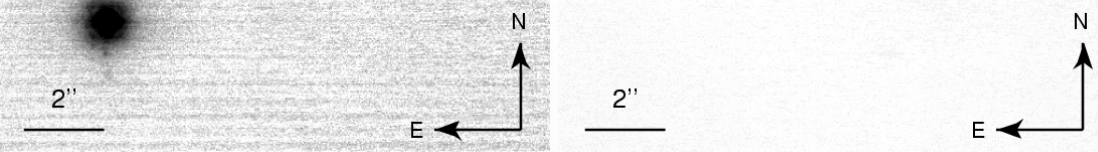

NB_1.64
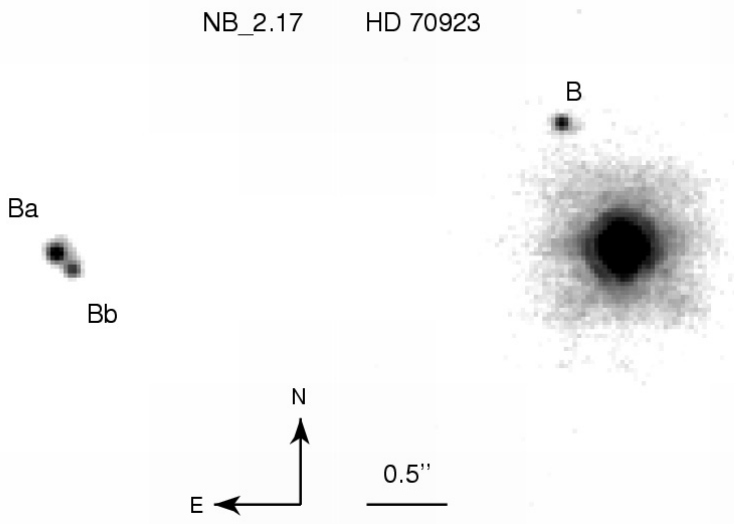

$0.5^{\prime \prime}$
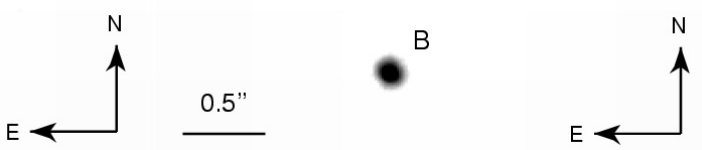

Fig. 4. NACO images of the true and likely bound companions. Note that the NB_1.64 filter produces several bright ghosts surrounding the image of the main target. 
A. Eggenberger et al.: The impact of stellar duplicity on planet occurrence and properties. I., Online Material p 11

HD 82241

NB 2.17 HD 104263

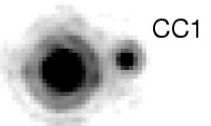

$0.5 "$

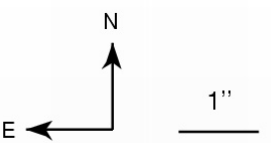

HD 134180

NB_2.17

HD 154682

B

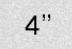

HD 196050

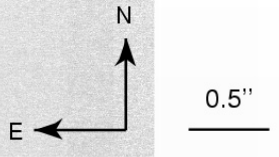

NB_2.17
HD 196050

$\mathrm{Ba}$

$\mathrm{Bb}$

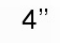

$4 "$

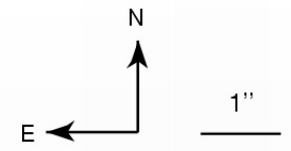

NB 2.17

HD 129642

$\mathrm{B}$

NB_1.64

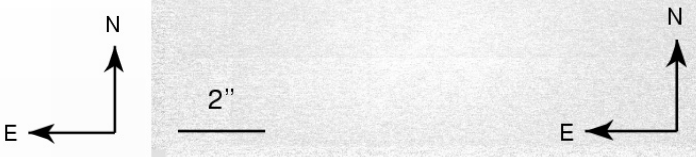

NB 2.17

HD 177830

NB_1.64

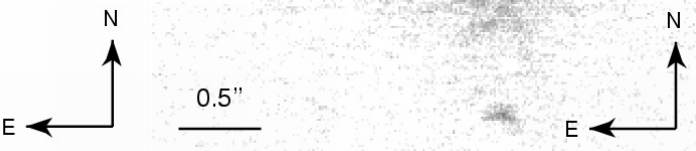

NB_2.17

HD 223913

NB_2.17

$\mathrm{Bb}$

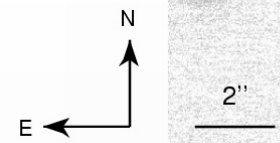

Fig. 4. continued. 